\title{
Vortices, Complex Flows and Inertial Particles
}

\author{
J. C. R. Hunt • R. Delfos · I. Eames • R. J. Perkins
}

Received: 25 April 2007 / Accepted: 4 June 2007 /

Published online: 23 August 2007

(C) Springer Science + Business Media B.V. 2007

\begin{abstract}
The properties of vortical structures at high Reynolds number in uniform flows and near rigid boundaries are reviewed. New properties are derived by analysing the dynamics of the main flow features and the related integral constraints, including the relations between mean swirl and bulk speed, the relative level of internal fluctuations to bulk properties, and connections between the steadiness and topology of the structures. A crucial property that determines energy dissipation and the transport of inertial particles (with finite fall speed) is the variation across the structure of the ratio of the mean strain rate $(\Sigma)$ to the mean vorticity $(\Omega)$. It is shown how, once such particles are entrained into the vortical regions of a coherent structure, they can be transported over significant distances even as the vortices grow and their internal structure is distorted by internal turbulence, swirling motions and the presence of rigid boundaries. However if the vortex is strongly distorted by a straining motion so that $\Sigma$ is greater than $\Omega$, the entrained particles are ejected quite rapidly. These mechanisms are consistent with previous studies of entrained and sedimenting particles in disperse two phase flows over flat surfaces, and over bluff obstacles and dunes. They are also tested in more detail here through laboratory
\end{abstract}

J. C. R. Hunt

Department of Space and Climate Physics, University College London,

Gower Street, London, WC1E 6BT, UK

R. Delfos

J. H. Burgers Centre, Delft University of Technology,

Leeghwaterstraat 21, 2628 CA, Delft, The Netherlands

I. Eames $(\bowtie)$

Department of Mechanical Engineering, University College London,

Torrington Place, London, WC1E 7JE, UK

e-mail: I_Eames@meng.ucl.ac.uk

R. J. Perkins

Laboratoire de Mécanique des Fluides et d'Acoustique, Université de Lyon, UMR CNRS 5509

École Centrale de Lyon, 36 avenue Guy de Collongue, 69134, Ecully, France 
observations and measurements of 50-200- $\mu \mathrm{m}$ particles entrained into circular and non-circular vortices moving first into still air and then onto rigid surfaces placed parallel and perpendicular to the direction of motion of the vortices.

Keywords Turbulence $\cdot$ Vortices $\cdot$ Deposition

\section{Introduction}

In many environmental and industrial flow processes small inertial particles are entrained into eddies and vortices $[9,13]$ and typically transported over significant distances before being expelled or falling out as the flow structures impact on rigid surfaces or obstacles placed in the flow. Because of the general properties of vortical flows, similar structures occur in many types of turbulent and unsteady flows. Vortical structures have a common property that they propagate forwards in stationary fluid as a result of their own vorticity distribution. The simplest and best understood examples are circular vortex rings which propagate without changing their shape. Non-circular structures oscillate as they propagate [29] or the vorticity may be diffused through the whole structure which becomes a turbulent puff. In practical problems, the movement of these flow structures is greatly affected by external flows, which may be turbulent, and by the kinematic and dynamic effects of rigid surfaces. The distortion of the vortex structures also greatly influences the movements of particles within them and leads to the particles being deposited onto the ground [30].

In this paper, these flow and particle transport mechanisms are reviewed in general terms using the theory of vortical structures and particle dynamics (in Sections 2 and 3). New laboratory airflow experiments are described (in Section 4) of laminar and turbulent vortical structures expelled from various orifice shapes. In some cases these structures impacted onto a rigid plane surface, and their sensitivity to external air currents was also studied. Subsequently observations and qualitative measurements were made of the transport of 50-200- $\mu \mathrm{m}$ particles released into the flow at the orifice over a distance of about $1 \mathrm{~m}$ from the orifice. The particles either settled out of the vortices or directly impacted onto a plane surface placed normal to the flow. Many previous studies on particles in vortical flows have focussed on turbulent flows such as jets [39] in which the vortices formed and moved randomly. Batchelor and Nitsche [5] and Gilbertson and Yates [21] examined the ejection of dense particles from vertical translating spherical vortices while Eames and Gilbertson [16] examined how particles outside the vortex are entrained and transported over a finite vertical distance. However in this paper we propose a broader understanding by relating the general characteristics of the flow kinematics to particle motion within isolated vortical structures.

This study was also motivated by the practical need to calculate two-phase flows where particles are entrained into isolated vortical structures such as line or ring vortices, which have a finite circulation around the vortical region. This process can be observed, for example, in flow over sand ripples; the flow separates at the crest of the ripple and vortices are generated in the shear layer downstream (see Fig. 1a). These entrain particles from the bed, which remain trapped inside the vortices and are transported by them, until they fall out; either because the vortex has decayed or because it impinges on another ripple downstream and 

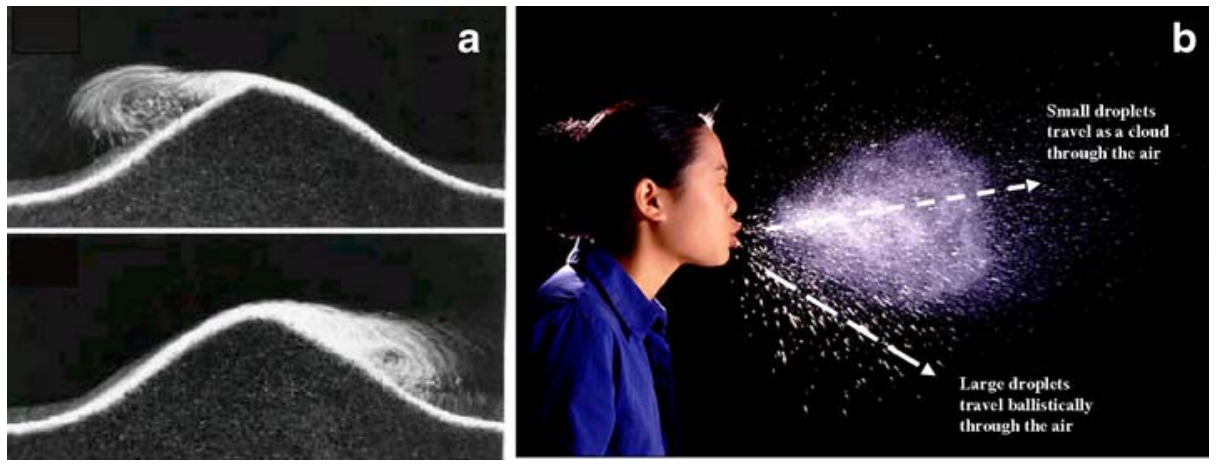

Fig. 1 a Vortices being shed by the oscillatory flow over a sand ridge, notice the clear region in the vortices (courtesy of Dr. Voropayev, A.S.U.) and b small and large droplets created by sneezing (taken from Tang et al. [44])

become sufficiently distorted to eject the particles. The particles are then deposited on the bed, where they contribute to the formation of other ripples. This process of vortex entrainment and deposition has been identified as a mechanism for the growth and persistence of ripples and dunes [3, 49]. A similar process has been exploited to improve the efficiency of the filtration of molten aluminium; surface roughness elements generate vortices which entrain the small solid particles and concentrate them in the core regions. This clustering of the small particles promotes agglomeration into larger particles which are then ejected from the vortices and deposited on the surface [1].

Biological and medical studies also involve considering how droplets and particles are transported in vortical structures generated, for instance, by coughing, breathing and sneezing (eg Hawthorne [22]). Both breathing and sneezing provide ample opportunity to generate droplets ladenwith bacteria and this is important for the transmission of some airborne diseases (see Fig. 1b and Tang et al. [44]). This process also featured in a recent court case in London [6]. The important issue was whether droplets of about 50-200 $\mu \mathrm{m}$ in diameter exhaled at $1 \mathrm{~m} / \mathrm{s}$ from a human mouth could travel a distance of about $0.5 \mathrm{~m}$, possibly in a turbulent environment with a weak cross-wind. Although it had been argued by the prosecution that such droplets could only travel about $0.2 \mathrm{~m}$, the analysis presented here (which greatly benefited from conversations with Frans Nieuwstadt) shows that vortical structures (ignored by the prosecution) even if quite imperfect and turbulent, can transport particles over significantly greater distances than uniform flow.

\section{Dynamics of Vortical Structures}

\subsection{Isolated structures}

Observations and theoretical arguments show that in high Reynolds number flows (with velocity $\boldsymbol{u}$ ), the vorticity $(\boldsymbol{\omega})$ tends to be concentrated in coherent structures or eddies [48]. These can be studied by approximating them as regions $\mathcal{D}_{\omega}$ with a 


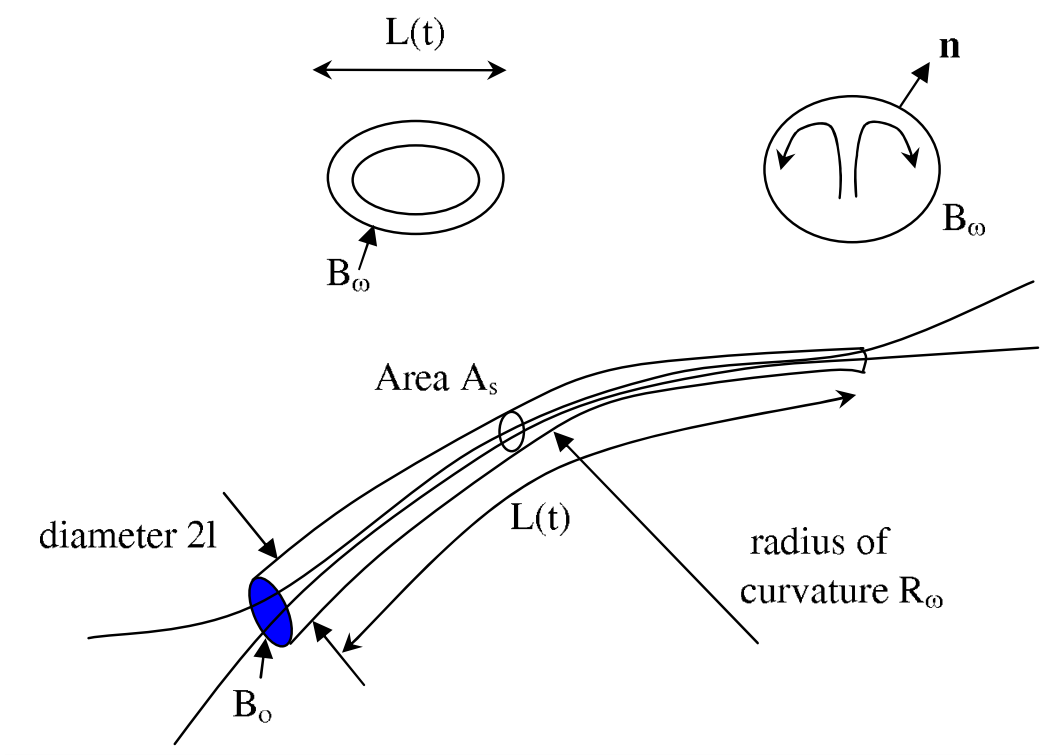

a

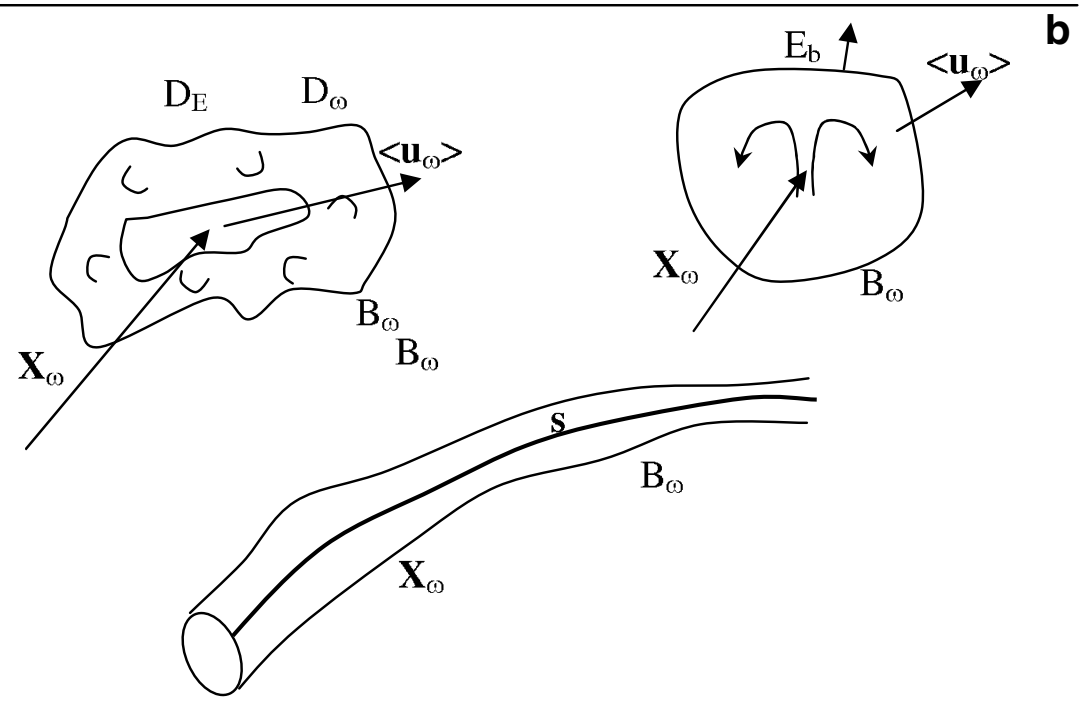

Fig. 2 Schematic showing a inviscid, idealised vortical structures and $\mathbf{b}$ turbulent vortical structures. $\boldsymbol{X}_{\omega}$ is the reference location of the structure and $E_{b}$ is the conditional average speed of the interface moving outwards through viscosity or turbulent entrainment. $L$ is a typical lengthscale of the vortex

bounding surface $\mathcal{B}_{\omega}$ outside which the vorticity is negligible (see Fig. 2). Some eddy structures are formed where vortex lines are concentrated within elongated regions of finite length (such as vortex tubes). Then $\mathcal{B}_{\omega}$ is an open surface on which there is a small region $\mathcal{B}_{o}$ where vortex lines enter and leave. The characteristics of these structures depend broadly on whether their shapes are elongated in the form of thin tubes and sheets with thickness $l$, or whether they are spheroidal or circular in the 
form of vortical lumps, puffs or rings. The typical mean square magnitude of the velocity and vorticity in these structures are $u_{\omega}$ and $\omega_{\omega}$. The initial value of $u_{\omega}$ is $u_{0}$. In all cases they are assumed to have finite overall length scales $L(t)$ where $L(0)=L_{0}$ $[4,23,41]$.

The topology or genus $g$ of the structures as defined by the geometrical form of $\mathcal{B}_{\omega}$ is usually no more complex than that corresponding to a sphere $(g=0)$ or a ring $(g=1)(\mathrm{eg}[18,26])$. The topology of the vortex lines within the structures on their surface may sometimes be quite complex $[2,7]$. Within these vortical structures the square of the mean square vorticity $\Omega^{2}=\omega_{i}^{2}$, where $\omega_{i}=\epsilon_{i j k} \partial u_{j} / \partial x_{k}$ in tensor notation, is comparable with or greater than the mean square strain rate $\left(\Sigma^{2}=\right.$ $\Sigma_{i j} \Sigma_{i j}$ where $\left.\Sigma_{i j}=\frac{1}{2}\left(u_{i, j}+u_{j, i}\right)\right)$ [25]. For high Reynolds number flows $(\operatorname{Re} \gg 1)$, $\Omega^{2} / \Sigma^{2} \ll 1$ outside $\mathcal{D}_{\omega}$, while $\Omega^{2} / \Sigma^{2} \leq 1$ inside the structure. Since $\nabla \cdot \omega=0$, the normal component of vorticity $\boldsymbol{\omega} \cdot \boldsymbol{n}$ is also zero outside the bounding surfaces $\mathcal{B}_{\omega}$, where $\boldsymbol{n}$ is the unit vector normal to the boundary $\mathcal{B}_{\omega}$. The discontinous form of the tangential component of vorticity $\omega_{s}=|\boldsymbol{n} \cdot(\boldsymbol{\omega} \times \boldsymbol{n})|$ is either defined by a finite step in vorticity $\Delta \Omega_{s}$, in which case $\omega_{s}=(1-H(n)) \Delta \Omega_{s}$, or it may have a singularity so that $\omega_{s}=\left|\Delta \boldsymbol{u}_{s} \times \boldsymbol{n}\right| \delta(n)$ if there is a finite jump $\Delta \boldsymbol{u}_{s}$ in the tangential component of velocity. Here $H(n), \delta(n)$ are the usual Heaviside step and delta functions [51]. For viscous laminar flows (at high $R e$ ) there is intense diffusion of vorticity from the surface $\mathcal{B}_{\omega}$ but this is generally confined to a viscous boundary or shear layer of thickness (in the normal direction) $l_{\mathcal{B}}$. For high $R e, l_{\mathcal{B}}$ is small compared with the overall scale $L$ of the structure. A recirculating vortical flow within an isolated structure is generally unstable to small amplitude disturbances (eg [36, 42]) so that the motions within $\mathcal{B}_{\omega}$ become turbulent. The bulk velocity $\left\langle\boldsymbol{u}_{\omega}\right\rangle$ and displacement $\boldsymbol{X}_{\omega}$ of the coherent structure are defined as

$$
\left\langle\boldsymbol{u}_{\omega}\right\rangle=\frac{1}{V_{\omega}} \int_{\mathcal{D}_{\omega}} \boldsymbol{u} \mathrm{d} V, \quad \boldsymbol{X}_{\omega}=\int_{0}^{t}\left\langle\boldsymbol{u}_{\omega}\right\rangle \mathrm{d} t .
$$

Although the shapes and even the topologies of isolated eddy structures change as they move, depending on their initial form, they have in common certain broad characteristic flow features and overall dynamics, with the magnitude of the motions determined by relevant invariant integral conditions.

\subsubsection{Fluid volume}

For incompressible inviscid flow, the volume $V_{\omega}$ of $\mathcal{D}_{\omega}$ remains constant, ie

$$
\frac{\mathrm{d} V_{\omega}}{\mathrm{d} t}=0 .
$$

Depending on its initial form and the velocity distribution in $\mathcal{D}_{\omega}$, the shape may not change significantly. However, for viscous and turbulent flows, as the thickness of the vortical region expands at a velocity $E_{b}$ (see Fig. 2), $V_{\omega}$ increases, ie

$$
\frac{\mathrm{d} V_{\omega}}{\mathrm{d} t} \sim E_{b} S_{\mathcal{B}}
$$

where $S_{\mathcal{B}}$ is the area of the bounding surface, $S_{\mathcal{B}} \sim 4 \pi L^{2}$ for a spheroid of radius $L$ or $S_{\mathcal{B}} \sim 2 \pi L l$ for a tube of radius $l$ and length $L$, and $E_{b}$ is the boundary entrainment velocity [46] which is of the order of the rms velocity $u_{0}$. The boundary entrainment 
coefficient $\alpha_{B}=E_{b} /\left|\left\langle\boldsymbol{u}_{\omega}\right\rangle\right|$ is quite sensitive to the shape of the structure, for example being smaller for ring vortices than for spheroidal puffs.

\subsubsection{Internal volume flux}

Consider a vortex tube with genus $g=1$ connected like a vortex ring, in which there is an 'axial' component $u_{s}=\boldsymbol{u} \cdot \boldsymbol{s}$ parallel to the axis of the tube, where $\boldsymbol{s}$ is the unit vector parallel to the vortex tube. Since $\mathcal{B}_{\omega}$ is a material surface in an inviscid flow there is a constraint on the volume flux $Q_{s}$ parallel to the tube, ie

$$
Q_{s}(s, t)=\int_{A_{s}} u_{s} \mathrm{~d} A,
$$

where $A_{s}$ is the cross-section area of the vortex tube, so that

$$
\frac{\mathrm{D} A_{s}}{\mathrm{D} t}=-\frac{\partial Q_{s}}{\partial s},
$$

where $s$ is the distance along the tube (see Fig. 2). Thus $Q_{s}$ is constant if the cross sectional area $A_{s}$ does not vary in time or spatially along the vortex tube. In a viscous flow, $\mathcal{B}_{\omega}$ is not a material surface, and (4) is only approximately valid.

\subsubsection{Circulation and helicity}

For an inviscid fluid, by Kelvin's theorem, the circulation $\Gamma(s, t)$ taken on any material loop $\mathcal{L}$ around a vortex tube is constant with time even if $A_{s}$ or $l$ varies with time, ie

$$
\frac{\mathrm{d} \Gamma(s, t)}{\mathrm{d} t}=\frac{\mathrm{d}}{\mathrm{d} t} \oint_{\mathcal{L}} \boldsymbol{u} \cdot \mathrm{d} \boldsymbol{s}=\frac{\mathrm{d}}{\mathrm{d} t} \int_{A_{s}} \boldsymbol{\omega} \cdot \mathrm{d} \boldsymbol{A}=0 .
$$

Equation 5 holds true for a vortex tube (genus 1) even if viscous stresses are significant within the shear layer surrounding the vortex. The classical description of the swirl velocity $u_{\theta}$ in a straight vortical tube is provided by Lamb [32]:

$$
u_{\theta}=\frac{\Gamma}{2 \pi r}\left(1-\exp \left(-\frac{r^{2}}{2 l^{2}}\right)\right)
$$

which is valid even when the radius grows through the diffusion of vorticity. However, for a vortical lump with a continuous and chaotic vorticity distribution within $\mathcal{B}_{\omega}$, the conservation of $\Gamma$ along fluid loops does not provide an integral constraint for the structure as a whole, when other integral constraints are relevant. The volume integral of vorticity is zero within $\mathcal{D}_{\omega}$, even when the shape and mean velocity are changing, the total helicity $H_{v}$ is constant, ie

$$
\int_{\mathcal{D}_{\omega}} \boldsymbol{\omega} \mathrm{d} V=0, \quad \text { and } \quad \frac{\mathrm{d} H_{v}}{\mathrm{~d} t}=\frac{\mathrm{d}}{\mathrm{d} t} \int_{\mathcal{D}_{\omega}} \boldsymbol{u} \cdot \boldsymbol{\omega} \mathrm{d} V=0 .
$$

For a vortex tube with a helical flow, ie $\boldsymbol{u} \cdot \boldsymbol{\omega} \neq 0$, there is a finite helicity flux $H_{v}^{A}=$ $\int_{A_{s}} \boldsymbol{u} \cdot \boldsymbol{\omega} \mathrm{d} A$. For example if a vortex ring with swirl is distorted (eg by reducing its circumference by a factor $\lambda$ as it passes through a contraction), then since $\omega_{\theta} \sim \lambda$ and the volume of the vortical tube $V_{\omega}$ is constant, $u \sim 1 / \lambda$. In other words the swirl velocity increases ( since $\lambda<1$ ). 


\subsubsection{Linear and angular momentum}

Vortical structures move and distort as a result of the flow induced by the nonuniform distribution of $\omega$ within $\mathcal{D}_{\omega}$. Vortex lines, with a curvature $R_{\omega}$, induce a velocity field $\boldsymbol{u}(\boldsymbol{x}, t)$ which extends outside $\mathcal{D}_{\omega}$. When the total vorticity in the whole flow domain $\mathcal{D}$ is zero $\left(\int_{D} \omega \mathrm{d} V=\mathbf{0}\right)$, the flow in the whole domain $\mathcal{D}$ may have a finite momentum integral or impulse,

$$
\boldsymbol{I}_{M}=\frac{1}{2} \rho \int_{\mathcal{D}} \boldsymbol{x} \times \omega \mathrm{d} V,
$$

for three-dimensional flows (for two-dimensional flows, the coefficient $1 / 2$ is dropped). For an isolated vortical structure $\boldsymbol{u}$ and $\boldsymbol{\omega}$ vary in space and time, but the impulse is constant even in a viscous flow, providing the flow is unbounded (as can be shown by integrating the equation of motion).

The impulse of a steadily propagating two-dimensional or axisymmetric vortex can be related to the mean velocity of propagation and vortex geometry through

$$
\boldsymbol{I}_{M}=\rho\left(1+C_{m}\right) V_{c}\left\langle\boldsymbol{u}_{\omega}\right\rangle
$$

where $V_{c}$ is the volume enclosed by the bounding streamlines and $C_{m}$ is a geometrical coefficient akin to (but different from) the added-mass coefficient of a rigid body. Expression (9) is sometimes used by experimentalists to estimate the impulse of a vortex, eg Stamhuis and Nauwelaerts [43] and Dabiri et al. [10].

The impulse associated with the fluid carried with the vortex is $V_{c}\left\langle\boldsymbol{u}_{\omega}\right\rangle$ while the impulse associated with the flow outside the vortex is $C_{m} V_{c}\left\langle\boldsymbol{u}_{\omega}\right\rangle$. The volume $V_{c}$ corresponds to the volume of matter transported with the vortex. For a point dipolar vortex consisting of cores of radius $l$ separated by a distance $L, V_{\omega}=2 \pi l^{2}$, $V_{c} \approx \pi L^{2}, C_{m} \approx 1$; for a Hill's spherical vortex of radius $L, V_{\omega}=4 \pi L^{3} / 3, V_{c}=V_{\omega}$ and $C_{m}=1 / 2$. For a vortex ring with curvature $R_{\omega}$ and core radius $l, V_{\omega} \approx 2 \pi^{2} R_{\omega} l^{2}$ and $I_{M}=\rho \pi R_{\omega}^{2} \Gamma$, so that $C_{m} V_{c}=4 \pi R_{\omega}^{3} /\left(\log \left(8 R_{\omega} / l\right)-1 / 4\right)$.

Other important conservation conditions can only be defined for certain geometries, for example, the total angular momentum $\boldsymbol{A}_{M}$, defined by

$$
\boldsymbol{A}_{M}=\frac{1}{3} \rho \int_{\mathcal{D}} \boldsymbol{x} \times(\boldsymbol{x} \times \boldsymbol{\omega}) \mathrm{d} V, \quad \text { and } \quad \frac{\mathrm{d} \boldsymbol{A}_{M}}{\mathrm{~d} t}=0,
$$

[4, p 520]. Thus, in the absence of body forces, the angular momentum of the vortex is conserved.

\subsubsection{Kinetic energy}

The total kinetic energy in the whole domain $\mathcal{D}$, denoted by $K E$, is defined as

$$
K E=\frac{1}{2} \rho \int_{\mathcal{D}} \boldsymbol{u} \cdot \boldsymbol{u} \mathrm{d} V
$$

In inviscid flows the kinetic energy of an isolated vortical structure is finite, either because $\boldsymbol{u} \cdot \boldsymbol{n}=0$ on an external boundary or because $|\boldsymbol{u}|$ decays sufficiently rapidly 
with distance from the centre of the structure $\left(|\boldsymbol{u}| \propto(r / L)^{-3}\right)$. Then in the absence of body forces $K E$ is conserved:

$$
\frac{\mathrm{d} K E}{\mathrm{~d} t}=0
$$

It is useful to decompose the contributions to the total kinetic energy of an eddy into the contributions from the internal motions $\left(K E_{\text {int }}\right)$ and the exterior flow $\left(K E_{\text {ext }}\right)$. If the vortex moves at an average velocity $\left\langle\boldsymbol{u}_{\omega}\right\rangle$,

$$
K E_{\text {int }}=\frac{1}{2} \rho \int_{D}\left(\boldsymbol{u}-\langle\boldsymbol{u}\rangle_{\omega}\right)^{2} \mathrm{~d} V, \quad K E_{\text {ext }}=\langle\boldsymbol{u}\rangle_{\omega} \cdot \boldsymbol{I}_{M},
$$

then

$$
\frac{\mathrm{d} K E}{\mathrm{~d} t}=\frac{\mathrm{d} K E_{\text {int }}}{\mathrm{d} t}+\frac{\mathrm{d} K E_{\text {ext }}}{\mathrm{d} t} .
$$

Even with no boundaries, the total kinetic energy decreases as a result of viscous dissipation:

$$
\frac{\mathrm{d} K E}{\mathrm{~d} t}=-\int_{\mathcal{D}} \epsilon(\boldsymbol{x}, t) \mathrm{d} V,
$$

where $\epsilon=-\mu \boldsymbol{u} \cdot \nabla^{2} \boldsymbol{u}$. The local dissipation $\epsilon$ may be negative or positive, especially as it varies rapidly from positive to negative in thin layers with high gradients of vorticity and velocity. Over the whole flow domain (if $|\boldsymbol{u}|,|\boldsymbol{\omega}| \rightarrow 0$ as $r \rightarrow \infty$ ),

$$
\int_{\mathcal{D}} \epsilon \mathrm{d} V=\mu \int_{\mathcal{D}} \omega^{2} \mathrm{~d} V+\mu \int_{\mathcal{S}} \boldsymbol{n} \cdot \boldsymbol{u} \times \omega \mathrm{d} S,
$$

[41, p 69] and the second term in (16) is zero and the total dissipation is

$$
\int_{\mathcal{D}} \epsilon \mathrm{d} V=\mu \int_{\mathcal{D}} \omega^{2} \mathrm{~d} V
$$

Note that the local variation of $\epsilon$ in the flow does not generally correspond to the variation of $\omega^{2}$. For a laminar flow characterised by the Reynolds number $R e_{v}=U_{\omega} l / v \gg 1$, the total dissipation is

$$
\int_{\mathcal{D}} \epsilon \mathrm{d} V \sim \mu \frac{U_{\omega}^{2}}{l^{2}} l L^{2} \sim \rho \frac{U_{\omega}^{3}}{L^{2}} L^{3} R e_{v}^{-1},
$$

where $U_{\omega}=\left|\left\langle\boldsymbol{u}_{\omega}\right\rangle\right|$. For a turbulent structure, small scale eddies lead to a much larger level of dissipation, which scales as $U_{\omega}^{3} L^{2}$.

\subsection{Integral constraints for vortical structures}

\subsubsection{Vortex tubes and rings}

As shown in Fig. 2, the vortex lines are clustered in a vortex tube (of core radius $l$ ). In general, tubes are not straight but have a curvature, $R_{\omega}$, which is typically of the same order as the vortex length $L$ and large compared with tube radius $l$. For a simple tube, the characteristic pattern of the relative magnitude of the vorticity and strain rate is defined by $\Omega^{2} / \Sigma^{2} \gg 1$ within the tube and $\Omega^{2} / \Sigma^{2} \ll 1$ outside the tube. For complex shapes of tubes (eg elliptical or even knotted, where $R_{\omega} \sim l$ ) $\Sigma^{2} / \Omega^{2}$ 
may be significant even within the tubes. Note that if there is flow along the tube, ie $Q \neq 0$, the vortex lines are twisted helically. In such a case the helicity integral $H_{v}$ has a non-zero constant value. If the tubes are in the form of isolated rings they have a closed boundary $\mathcal{B}_{\omega}$ around which the circulation $\Gamma$ is constant.

Let $\boldsymbol{b}$ be the binormal vector perpendicular to the plane of the curvature and to the axis of the vortex $\boldsymbol{s}$. The vortex curvature creates a self-induced flow, which causes the vortex to move with a component parallel to the direction of $\boldsymbol{b}$ determined by the Biot-Savart law [4]. As a result this local region of the flow makes a contribution to the impulse $\boldsymbol{I}_{M}$ in the direction parallel to $\boldsymbol{b}$. The magnitude of $\left\langle\boldsymbol{u}_{\omega}\right\rangle \cdot \boldsymbol{b}$ is inversely proportional to $R_{\omega}$ but is also weakly dependent on the core radius $l$. Around an idealised ring vortex with no swirl, the curvature is constant, so that in a static fluid, it moves steadily along its central axis with velocity

$$
U_{\omega}=\left\langle\boldsymbol{u}_{\omega}\right\rangle \cdot \boldsymbol{b}=\frac{\Gamma}{4 \pi R_{\omega}}\left(\log \left(\frac{8 R_{\omega}}{l}\right)-\frac{1}{4}\right) .
$$

The impulse and kinetic energy associated with the vortex ring are

$$
\boldsymbol{I}_{M}=\rho \pi \Gamma R_{\omega}^{2} \boldsymbol{b}, \quad K E=\frac{1}{2} \rho \Gamma^{2} R_{\omega}\left(\log \left(\frac{8 R_{\omega}}{l}\right)-\frac{7}{4}\right)
$$

$[41,45]$. Motions parallel to the vortex tube $u_{s}=\boldsymbol{u} \cdot \boldsymbol{s}$ can also occur in a curved vortex tubes. The radial inertial force $u_{s}^{2} / R_{\omega}$ is either balanced by the unsteady radial growth of the ring or by a radial external pressure gradient (for inviscid flow) $\nabla p$ in the irrotational flow outside $\mathcal{D}_{\omega}$ for example when a ring vortex is generated with swirl in a converging flow. Experiments show that swirling or helical vortex rings move quite unsteadily [40]. Elliptical vortex rings however are always unsteady because the radius of curvature varies along the tube, being smallest at the sharp ends and largest in the middle. Consequently these vortex tubes deform and oscillate as they move because the tube moves faster at the sharp ends which move out of the plane of the ring, causing complex oscillatory bending around the ring on a time scale of order $R_{\omega}^{2} / \Gamma \log \left(R_{\omega} / l\right)$ (eg [29]). Figure 3 shows numerical calculations of an oscillating elliptical vortex ring showing the oscillating nature of the vortex motion.
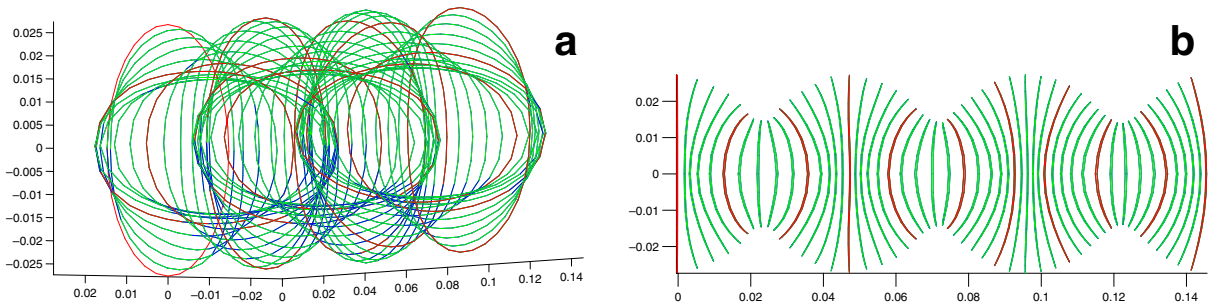

Fig. 3 Simulated elliptical vortex ring, moving from left to right where a and $\mathbf{b}$ show respectively angled and side views. On its path, initially the highly curved top and bottom part travel forward with the highest speed, thereby losing curvature; at the same time folding the side parts of the ring and increasing their curvature. As a result, the roles reverse; the sides increase speed, and pick up and overtake the top and bottom part, and so it continues 
Despite these irregular motions, the vortex continues to move in the same direction as it did initially because $\boldsymbol{I}_{M}$ is conserved. Since a significant component of the kinetic energy of the vortex is now associated with the oscillations and $\boldsymbol{I}_{M}$ is constant, it follows from (13) that the average speed $U_{\omega}$ of the vortex is reduced. The same mechanism causes curved vortex tubes that typically occur in turbulent and complex flows to deform, oscillate and sometimes reconnect into ring vortices. The effect of viscosity when $R e_{v}=\Gamma / v \gg 1$ does not change these results qualitatively over the inertial vortex timescale $T_{I} \sim R_{\omega} / U_{\omega}$, which is also the time taken for the vortex to move a distance of order $R_{\omega}$ and to undergo distortion. However, over greater time scales other qualitative effects also occur. Firstly, for laminar flows, during this period the thickness of the vortex tube is slightly increased by order $\left(v T_{I}\right)^{\frac{1}{2}} \sim l R e_{v}^{-\frac{1}{2}}$, but as the tube moves with velocity $\left\langle\boldsymbol{u}_{\omega}\right\rangle$ the vorticity field is slightly deformed and diffuses into a thin 'wake' (with thickness $l_{w}$ ). The straining flow field around the moving vortex ring of radius $R_{\omega}$ limits the growth of the wake thickness [24], to a value

$$
l_{w} \sim\left(\frac{v l}{\left|\left\langle\boldsymbol{u}_{\omega}\right\rangle\right|}\right)^{\frac{1}{2}} \sim \frac{R e_{v}^{-\frac{1}{2}} l}{\left(\log \left(R_{\omega} / l\right)\right)^{\frac{1}{2}}} .
$$

Thus $l_{w}$ is much less than the outer radius of the vortex ring $R_{\omega}$ (eg [47]). The converging flow in the near wake of a vortical structure, causes vorticity on one side of the wake to diffuse into vorticity of the opposite sign on the other side of the wake. This effective annihilation of wake vorticity means that negligible vorticity is left behind as the vortex moves. For turbulent dipolar vortices, virtually no wake vorticity is deposited because it is entrained over the rear of a moving vortex (see Flor [15]). For turbulent vortex rings, a fraction of the vorticity is detrained from [50]. Consequently the dynamics of moving vortical structures in complex flows can be considered in isolation due to the rapid decay of the flow perturbation. There may be a background flow field generated by the other eddies. Note that when vortices are generated by the injection of a volume of fluid much larger than $V_{c}$, a great deal of the excess fluid is lost during its adjustment phase.

The second critical effect of viscosity is also associated with the cancellation of opposite sign vorticity when elements of oscillating vortex tubes collide with each other. This enables new vortex rings to be formed, which can separate from each other, transporting kinetic energy away from the original location of the vortex tube. But the impulse is not changed by such significant topological changes.

The third general effect occurring over the time scale $T_{I} R e_{v}$ is caused by viscous stresses acting over the radius of the ring. Damping of oscillations and the smoothing of paths of vortex lines eventually causes vortex rings of any initial shape to form into spheroidal vortex rings.

\subsubsection{Vortical puffs or fluid lumps}

In spheroidal vortical structures (such as puffs or fluid lumps) with genus $g=0$ and bounding surface $\mathcal{B}_{\omega}$ the major and minor axes of the structure are typically of similar magnitude $L$, and the strain rate is also significant in the puff with $\Omega^{2} / \Sigma^{2} \sim 1$. As with vortex tubes, their behaviour depends on the type of eddy motion and also on the relative magnitude of the impulse and the total kinetic energy $K E$. This ratio is 
defined by $\widetilde{K E_{\text {ext }}}=K E_{\text {ext }} / K E$ (ie $\left\langle\boldsymbol{u}_{\omega}\right\rangle \cdot \boldsymbol{I}_{M} / K E$ ). The relative magnitude $\tilde{H}$ of the helicity integral to the $K E$, is also a defining property where $\tilde{H}=H_{v} L / K E$.

When $\widetilde{K E}_{\text {ext }} \sim 1$ and $|\tilde{H}| \ll 1$, the eddy can move steadily under its own induction (like Hill's spherical vortex [4]). When turbulent entrainment is important, the volume of the vortex puff $V_{\omega}\left(\approx V_{c}\right)$ grows according to (3). Approximating $V_{\omega} \sim \frac{4}{3} \pi L^{3}$ and the impulse by $\boldsymbol{I}_{M} \sim\left(1+C_{m}\right) V_{c} U_{\omega} \boldsymbol{b}$, then the radius of the puff grows as

$$
L \sim L_{0}\left(1+\frac{12 \alpha_{B} U_{\omega}(t=0) t}{L_{0}}\right)^{\frac{1}{4}}
$$

Since impulse is conserved, then the velocity of the coherent structure decays approximately as

$$
\left\langle\boldsymbol{u}_{\omega}\right\rangle \sim\left\langle\boldsymbol{u}_{\omega}\right\rangle(t=0)\left(1+\frac{12 \alpha_{B} U_{\omega}(t=0) t}{L_{0}}\right)^{-\frac{3}{4}},
$$

where $\alpha_{B} \approx 0.1$ (see [42]).

When $\widetilde{K E}$ ext $\ll 1$ or if $|\tilde{H}| \sim 1$ (eg two vortex rings are positioned opposite to each other or an ellipsoidal vortex with elliptical vortex lines, or a swirling spherical vortex), the internal flow is primarily an unsteady motion, and the largest diameter $L$ increases as a result of the twisted vortex lines inducing motion in all directions. When $u_{0} \gg U_{\omega}$, the vortex grows in size due to the self induced motion of the vortex lines with $L \sim u_{0} t$. Since the kinetic energy is largely determined by the internal motion within the vortex and is approximately conserved, then $u_{0} \sim(K E / \rho)^{\frac{1}{5}} t^{-3 / 5}$ and $L \sim(K E / \rho)^{\frac{1}{5}} t^{2 / 5}$. The mean speed then decreases as $U_{\omega} \sim U_{\omega}(0)(K E / \rho)^{-3 / 5} t^{-6 / 5}$. This shows that as unsteady oscillations within a vortical eddy grow, its advective speed $U_{\omega}$ decreases faster than the fluctuations $u_{0}$ over a period of order $L / u_{0}$. In general such eddies are rotating and non-spherical so that they have finite angular momentum $\boldsymbol{A}_{M}$. As is commonly observed the average swirl velocity $\left\langle\boldsymbol{u}_{S}\right\rangle$ decreases faster $\left(\sim t^{-8 / 5}\right)$ than the advective speed $U_{\omega}$ and the rms fluctuations $u_{0}$. The relatively fast decay of advective and swirl velocities may be one reason why the decay of fluctuations in turbulent eddies is not sensitive to the initial form of the eddy motion [11].

Vortex rings and puffs become turbulent when the Reynolds number is high enough. Depending on the level of external velocity fluctuations $\left(u_{E} / u_{0}\right)$ and on the form of the vortical structure (eg elliptical or circular) the critical Reynolds number is reduced and the level of fluctuations in the structure $\left(u_{0} / U_{\omega}\right)$ can be amplified. However the sharp gradients of turbulence at the bounding surfaces $\mathcal{B}_{\omega}$ of these structures amplify the mean vorticity near $\mathcal{B}_{\omega}$ and ensure that (provided $u_{E} / u_{0} \leq 1$ ) the boundary interface is approximately continuous and does not naturally break up into smaller structures (eg Hunt et al. [28], Westerweel et al. [51]).

\subsection{Interactions with resistive surfaces}

There are two main types of mutual interactions between vortical structures and plane resistive surfaces (where $\boldsymbol{u} \cdot \boldsymbol{n}=\mathbf{0}$ ) at high Reynolds numbers according to 
whether the initial impulse is (1) parallel to the surface $\left(\boldsymbol{I}_{M} \cdot \boldsymbol{n}=0\right)$ or (2) perpendicular to the surface $\left(\boldsymbol{I}_{M} \times \boldsymbol{n}=\mathbf{0}\right)$. The interaction of a vortical structure and a plane surface is through kinematic 'blocking' by the boundaries and the dynamic effect through the no-slip condition, acting as a source of vorticity. From the invariants of Sections 2.1 and 2.2, the main features of the interaction can be defined mathematically and physically at high Reynolds numbers when viscous stresses can be neglected. These studies are provide insight into interactions between vortical structures.

The qualitative effects of a plane surface on the vorticity $\boldsymbol{\omega}^{(0)}(\boldsymbol{x}, t)$ of the flow (for $n>0$ ) can be studied by analysing the image vorticity field $\omega^{(I)}(\boldsymbol{x}, t)$ (for $n<0$ ). The irrotational velocity field $\boldsymbol{u}^{(I)}$ induced by $\boldsymbol{\omega}^{(I)}($ in $n<0)$ satisfies the kinematic condition $\boldsymbol{u}^{(I)} \cdot \boldsymbol{n}=-\boldsymbol{u}^{(0)} \cdot \boldsymbol{n}$, where $\boldsymbol{u}^{(0)}$ is the velocity field induced by $\boldsymbol{\omega}^{(0)}$. Thus $\boldsymbol{u}=\boldsymbol{u}^{(0)}+\boldsymbol{u}^{(I)}$. Note that although $\boldsymbol{\omega}^{(0)}$ is affected by the image flow field $\boldsymbol{u}^{(I)}$, the vorticity of fluid elements can be amplified or reduced but only change sign in inviscid flows if the fluid elements change direction, which does not occur for vortex structures with simple shapes.

\subsubsection{Impulse parallel to the surface}

A typical example in this case is where a vortex ring or puff moves in the $x$-direction parallel to a surface, ie $\boldsymbol{I}_{M} \cdot \boldsymbol{n}=\mathbf{0}$. The flow field of the image vorticity bends the vortex tubes so that the distorted vortex deflects towards the surface inducing vertical fluctuations and also the whole vortex spreads out so that $L$ increases. We now apply the conservation condition of Section 2.1 and the analysis of Section 2.2. It follows that, as for an isolated vortex puff, since $\boldsymbol{I}_{M} \times \boldsymbol{n}$ is conserved and since $L$ and $K E_{\text {int }}$ increase, $\left\langle\boldsymbol{u}_{\omega}\right\rangle \times \boldsymbol{n} / u_{0}$ decreases at approximately the same rate as for a nonsymmetric vortical structure. This is consistent with experiments on vortices and puffs along a surface (Section 4). The distortion of the vortex develops an impulse in the velocity field with a vertical component ie $\boldsymbol{I}_{M} \cdot \boldsymbol{n} \neq 0$. But since no net vertical force is applied to the flow, this is balanced by a vertical force $\boldsymbol{F}$ exerted by pressure on the rigid surface, ie $\boldsymbol{F}=\int p(n=0) \boldsymbol{n} \mathrm{d} A=-\left(\boldsymbol{I}_{M} \cdot \boldsymbol{n}\right) \boldsymbol{n}$. Near the surface the vortex lines are bent and there is an increase in the strain rate $\Sigma$ relative to the vorticity $\Omega$ which is important for particle dispersion.

\subsubsection{Impulse perpendicular to the surface}

When a vortical structure moves towards a rigid surface, the image vorticity field induces stretching of the vortex lines, a large increase in the radius $L$ and the velocity components parallel to the surface. As the structure approaches the surface the normal component $\left\langle\boldsymbol{u}_{\omega}\right\rangle \cdot \boldsymbol{n} \rightarrow 0$ and $\boldsymbol{I}_{M}(t) / \boldsymbol{I}_{M}(t=0) \rightarrow 0$. The impulse causes a reactive force at the surface.

It is not possible for any type of vortical structure in a homogeneous fluid to 'bounce' or be reflected off the surface. Since $K E$ is constant in inviscid or high $R e$ flows, the perturbation velocity $u_{0}$ becomes much larger than the mean velocity $\left\langle\boldsymbol{u}_{\omega}\right\rangle$ over a period of order $L_{0} / u_{0}$. The opposing effect of the image vortex produces a large strain rate $\Sigma$ throughout the structure, even where vorticity is maximum. This is because as the vortical region is flattened, $\Sigma / \Omega$ increases. Because the radius $l(t)$ is so reduced by the distortion near the surface, this type of vortex-surface interaction is in practice greatly influenced by viscosity. In fact $l(t)$ becomes of the same order as the thickness of the viscous boundary layer $\left(\sim L R e_{v}^{-1 / 2}\right.$ for laminar flows). 


\subsubsection{Vortical structures advected through complex flows}

In many flows, vortical structures are advected through larger scale flows where the velocity gradients can change their strength, form and orientation, including the ratio $\Sigma / \Omega$. For example, certain integral properties are also changed, such as impulse, kinetic energy, but others such as the helicity integral (if the external flow is irrotational) may be invariant. Examples are considered in Section 3 with particle dynamics.

\section{Particle Dynamics in Vortex Structures}

Having identified the general properties of the flow in vortex tubes and eddies, it is now possible to describe how small inertial particles behave in these structures and under which circumstances they are expelled as a result of their inertial and buoyancy forces. The governing equation is derived for the displacement $\boldsymbol{X}=(X, Y, Z)$ of a small inertial particle with terminal fall speed $v_{T}$ and response time $t_{p}$. The equation of motion of a dense particle moving with velocity $v$ in a fluid with local velocity $\boldsymbol{u}(\boldsymbol{x}, t)$, is

$$
\frac{\mathrm{d} \boldsymbol{v}}{\mathrm{d} t}=\frac{1}{t_{p}}\left(\boldsymbol{u}-\boldsymbol{v}-v_{T} \hat{\boldsymbol{z}}\right), \quad \frac{\mathrm{d} \boldsymbol{X}}{\mathrm{d} t}=\boldsymbol{v},
$$

where for spherical particles of diameter $d_{p}$, the response time is $t_{p}=d_{p}^{2}\left(\rho_{p} / 18 \mu\right)$, and $v_{T}=g t_{p}$. For an earlier review of the particle transport phenomena in turbulence and key dimensionless variables and justification of (24), see Hunt et al. [27].

One of the keys to understanding the movement of particles in unsteady complex flows is to study the critical points in the particle trajectories, principally particle stagnation points (PSP). Davila and Hunt [13] and Eames and Gilbertson [16] studied the influence of critical points on the average settling velocity and dispersivity of particles settling near stationary and moving coherent structures and the geometry of the shadow regions. More recently, Chen et al. [8] have begun to analyse the spatial and temporal statistical distribution of these points in KS and DNS simulations of the flow field.

\subsection{Particle motion near vortex tubes}

In the first instance we consider the example of dense particles falling near a horizontal vortical tube.

\subsubsection{Particles in the core}

In this first example, the flow $\boldsymbol{u}$ is two-dimensional and described by the streamfunction, $\psi_{f}$, where

$$
\boldsymbol{u}=\left(-\frac{\partial \psi_{f}}{\partial z}, \frac{\partial \psi_{f}}{\partial x}\right)
$$


Here we are concerned with the flow within the vortex which is affected by an external straining flow, and described by

$$
\psi_{f}(x, z)=\frac{1}{2} \Omega\left(x^{2}+z^{2}\right)-\Sigma\left(\frac{1}{2} \sin 2 \gamma\left(z^{2}-x^{2}\right)+x z \cos 2 \gamma\right),
$$

where $\gamma$ is the inclination of the strain field to the horizontal axis. Particle dynamics are characterised by a Stokes number, $S_{t}=t_{p} /\left(\Omega^{2}+\Sigma^{2}\right)^{\frac{1}{2}}$. For $S_{t}=0$, it follows from (24) that the particles fall relative to the local fluid velocity so that

$$
\boldsymbol{v}=\boldsymbol{u}-v_{T} \hat{\boldsymbol{z}} .
$$

Under these conditions, the particle paths can be described by a particle streamfunction that is simply related to the flow streamlines by

$$
\psi_{p}=\psi_{f}-v_{T} x
$$

The critical point in the flow, where the particle velocity is zero, has coordinates

$$
X_{c}=-\frac{v_{T}(\Omega+\sin 2 \gamma \Sigma)}{\Omega^{2}-\Sigma^{2}}, \quad Z_{c}=\frac{\Sigma v_{T} \cos 2 \gamma}{\Omega^{2}-\Sigma^{2}} .
$$

A critical point cannot exist if $\Omega=\Sigma$, when the flow is a locally rectilinear shear flow. For $\Omega>\Sigma$, the particle paths are elliptical with an aspect ratio of $((\Omega-\Sigma) /$ $(\Omega+\Sigma))^{\frac{1}{2}}$ and the critical point is a stable centre, while for $\Omega<\Sigma$ the particle paths are hyperbolae and the critical point is unstable.

For finite $S_{t}$, the particle acceleration needs to be taken into account. For $\gamma=0$, the normalised equations for $X, Z$ are

$$
\begin{aligned}
& \ddot{\tilde{X}}=-\tilde{\Omega} \tilde{Z}+\tilde{\Sigma} \tilde{X}-\dot{\tilde{X}} \\
& \ddot{\tilde{Z}}=\tilde{\Omega} \tilde{X}-\tilde{\Sigma} \tilde{Z}-\dot{\tilde{Z}}
\end{aligned}
$$

where derivatives are taken with respect to $\tilde{t}=t / t_{p}, \tilde{\Omega}=\Omega t_{p}, \tilde{\Sigma}=\Sigma t_{p}$, and

$$
\tilde{Z}=Z-Z_{c}, \quad \tilde{X}=X-X_{c} .
$$

The basic solutions to the linear system $(30,31)$ are exponential, ie

$$
\tilde{X}=\hat{X} \exp (i \sigma \tilde{t}), \quad \tilde{Z}=\hat{Z} \exp (i \sigma \tilde{t}),
$$

where the dispersion relation is

$$
\tilde{\sigma}^{4}+\left(\tilde{\Omega}^{2}-\tilde{\Sigma}^{2}\right)-2 i \sigma^{3}-\sigma=0 .
$$

For particles with small inertia, $|\tilde{\Omega}|,|\tilde{\Sigma}| \ll 1, \sigma=\sigma_{0}+\epsilon$ where $\sigma_{0}=\left(\tilde{\Omega}^{2}-\tilde{\Sigma}^{2}\right)^{\frac{1}{2}}$ and $\epsilon=-i\left(\tilde{\Omega}^{2}-\tilde{\Sigma}^{2}\right)$. Therefore, for $\Omega^{2} / \Sigma^{2}>1$ the leading order term in the solution for inertial particles consists of elliptical trajectories around the critical point at a frequency $\sigma_{0} / t_{p}$ which depends only on the vorticity $\Omega$ and the strain rate $\Sigma$. To the next approximation, the particle orbits expand exponentially so that over a time $t_{e j}$ the particles escape from the vortex. Particles located a distance $R_{0}$ from the stable centre are ejected from a vortex of radius $l$ in time

$$
t_{e j} \sim \frac{\log \left(l / R_{0}\right)}{\left(\Omega^{2}-\Sigma^{2}\right) t_{p}} .
$$



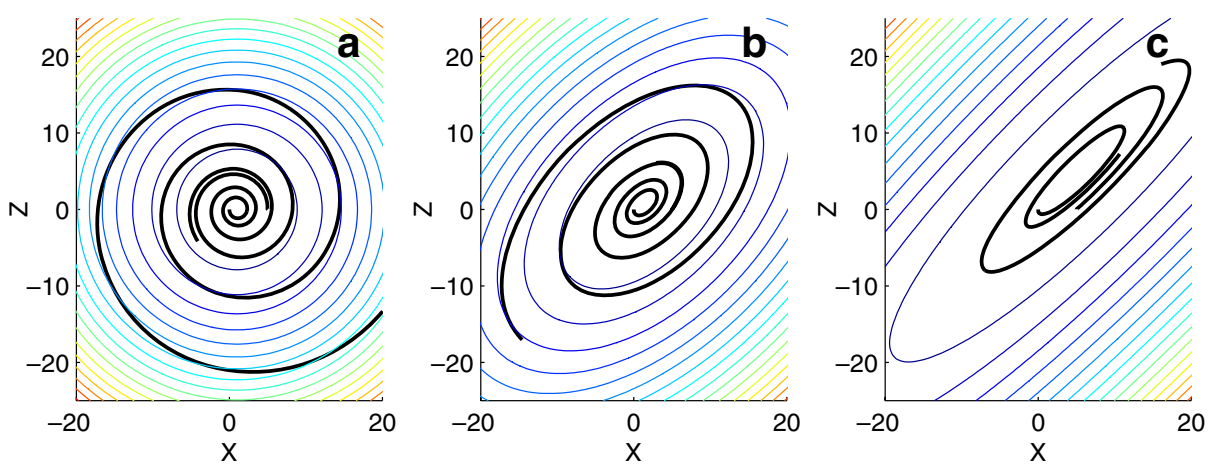

Fig. 4 Trajectories of two particles released near a strained vortex core. From a-c, the ratio of vorticity to strain is $\Omega / \Sigma=1 / 0,1 / 0.5,1 / 0.9$ and $\gamma=0$. The trajectories are plotted as a dark line over the time range $\tilde{t}=0$ to 20 , with $v_{T}=1, t_{p}=0.1$

An alternative view is to examine the radial equation of motion of the particle, which for small $S_{t} \ll 1$ reduces to a balance between the radial drag force and centrifugal force (ie $\dot{R} / t_{p} \sim\left(\Omega^{2}-\Sigma^{2}\right) R$ ), so that $R \sim R_{0} \exp \left(\int_{0}^{t} t_{p}\left(\Omega^{2}-\Sigma^{2}\right) \mathrm{d} t\right)$, which is equivalent to (35). Thus the smaller the particle response time and the closer to the critical point, the longer it takes for particles to be ejected from the vortex.

Figure 4 shows the trajectories of particles released near the centre of the strained vortex. As the strain rate increases, the average radial distance moved by the particles decreases progressively, increasing the particle residence time. This effect may be of importance for particles remaining within the strained vortices shed from ripples, shown in Fig. 1a.

\subsubsection{Particles outside the vortex core}

The vortex tube is moving horizontally with speed $U_{\omega}$ (from right-to left) and consists of a concentrated region of vorticity. In the frame moving with the vortex, the flow is azimuthal. A suitable model for the flow field is given by (6) (Lamb's vortex) - this is also the similarity solution studied by Marcu et al. [37] for dense particles moving near a Burgers vortex (where there is an external irrotational straining flow). The streamfunction corresponding to a fixed Rankine vortex (6) is

$$
\psi_{f}(x, y)=\frac{\Gamma}{2 \pi}\left[\log r+\frac{1}{2} E_{1}\left(\frac{r^{2}}{2 l^{2}}\right)\right],
$$

where $E_{1}$ is the exponential integral. The maximum azimuthal speed of the vortex is $U_{m} \sim \Gamma / 2 \pi l$. The usual Stokes number of the particles is $S_{t}=t_{p} U_{m} / l$. For $S_{t}=0$, we can define a particle streamfunction

$$
\psi_{p}=\psi_{f}+U_{\omega} z+v_{T} x=\frac{\Gamma}{2 \pi}\left[\log r+\frac{1}{2} E_{1}\left(\frac{r^{2}}{2 l^{2}}\right)\right]-U_{\omega} z-v_{T} x,
$$

where $U_{\omega}$ is the horizontal vortex speed. Figure 5 shows the topology of the particle streamlines for $v_{T} / U_{m}=0.01,0.2,5$ and positive circulation. For $v_{T} / U_{m}<1$ there are two critical points: a stable centre located at $x \sim 4 \pi v_{T} l^{2} / \Gamma$ (from (25)) and a saddle point located at $R_{p s p} \sim \Gamma / 2 \pi v_{T}$. A separatrix is created which encapsulates 

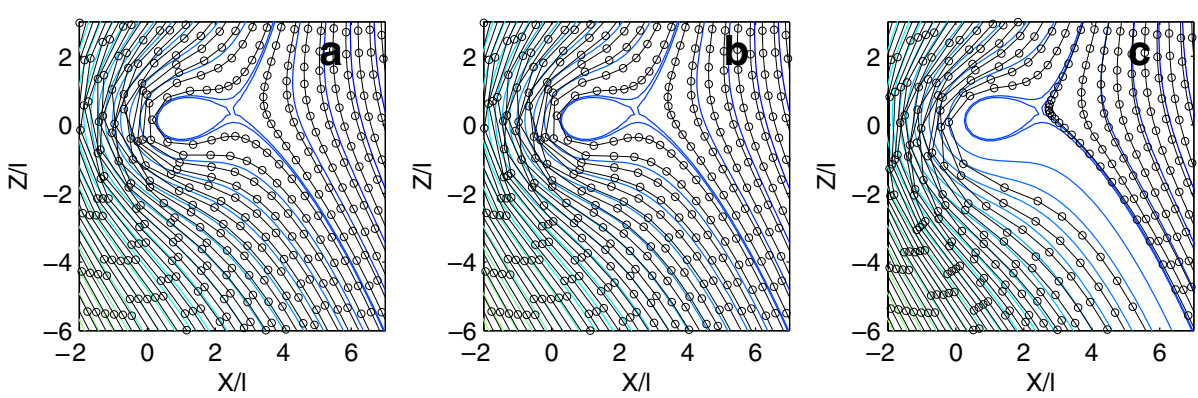

Fig. 5 Particles trajectories sedimenting past a Rankine vortex moving with at a constant speed along the $x$-axis. The trajectories are plotted in the frame moving with the vortex. The vortex speed is fixed at $U_{\omega}=0.01$, with $\Gamma=1, l=1$. The 3 panels correspond to a $v_{T} / U_{m}=0.01, \mathbf{b} 0.2$ and $\mathbf{c} 5$, respectively

the vortex. The saddle point is usually referred to as the PSP and essentially controls the bulk settling properties of the particles because the particle residence time increases with the logarithm of the initial distance from the stagnation streamline.

The characteristics of the movement of particles around the vortex can be defined by a local value of the modified Stokes number

$$
S_{t}^{*}=v_{T} t_{p} /\left(\Gamma / v_{T}\right)=\left(v_{T} / U_{m}\right)^{2} S_{t},
$$

$[13,37]$, which is based on an advective timescale characterised by the size of the shadow region. If $S_{t}^{*} \leq 1$ and $v_{T}<U_{m}$ the inertia of the particle causes the value of $R_{p m x}$ to increase by $\Delta R_{p m x} \sim-\tau_{p}^{2} \mathrm{~d} V / \mathrm{d} t \sim-\tau_{p} v_{T}$. The trajectories of all the particles on the left-hand side of the PSP progressively move further from the vortex so that they do not get swept back towards the lower side of the PSP. This is why they descend faster. Similarly particles on the upward moving (or right-hand) side of PSP are deflected further from the vortex where the upward velocity is weaker. These also descend faster than inertia-less particles. However when $S t^{*} \geq 1$ and $v_{T}>U_{m}$, the particles to the left of the PSP simply descend past the right-hand side of the vortex and do not pass around it. There is no longer an excluded shadow region for settling particles.
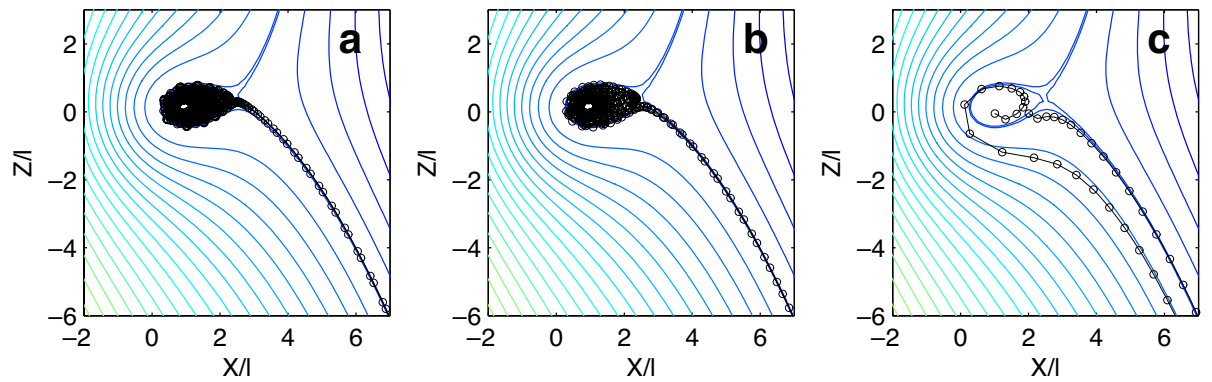

Fig. 6 Trajectories of particles released from inside a moving Rankine vortex. The parameters are the same as in Fig. 5 
Figure 6 shows the trajectories of dense particles released from within a steadily moving Rankine vortex in the vortex frame of reference. As the vortex moves with speed $U_{\omega}$ to the left, particles starting in the vortex initially move with the vortex before being ejected from the core. A particle starting a distance $R_{0}$ from the core centre is effectively transported a distance

$$
X_{d} \sim-U_{\omega} t_{e j}=\frac{U_{\omega} \log \left(R_{0} / R_{p s p}\right)}{\left(\Omega^{2}-\Sigma^{2}\right) t_{p}}=\frac{U_{\omega}\left(\log \left(2 \pi v_{T} l / \Gamma\right)+\log \left(R_{0} / l\right)\right)}{\left(\Omega^{2}-\Sigma^{2}\right) t_{p}},
$$

where $R_{0}$ is the initial distance from the centre. Since the particles are initially released along a line through the core of the vortex, the deposition is anticipated to decay exponentially with distance. Figure 7 shows the deposition profile for particles which are released with uniform spacing along a line starting from the centre and extending to the saddle point. The distance over which the particles are permanently transported, $X_{d}$, is normalised according to $X_{d} t_{p}\left(\Gamma / 4 \pi l^{2}\right)^{2} / U_{\omega}$ and is plotted as a probability density function for $v_{t} / U_{\omega}=0.1$ and 0.2 . As $S_{t}$ decreases, the curves collapses on to an exponential curve.

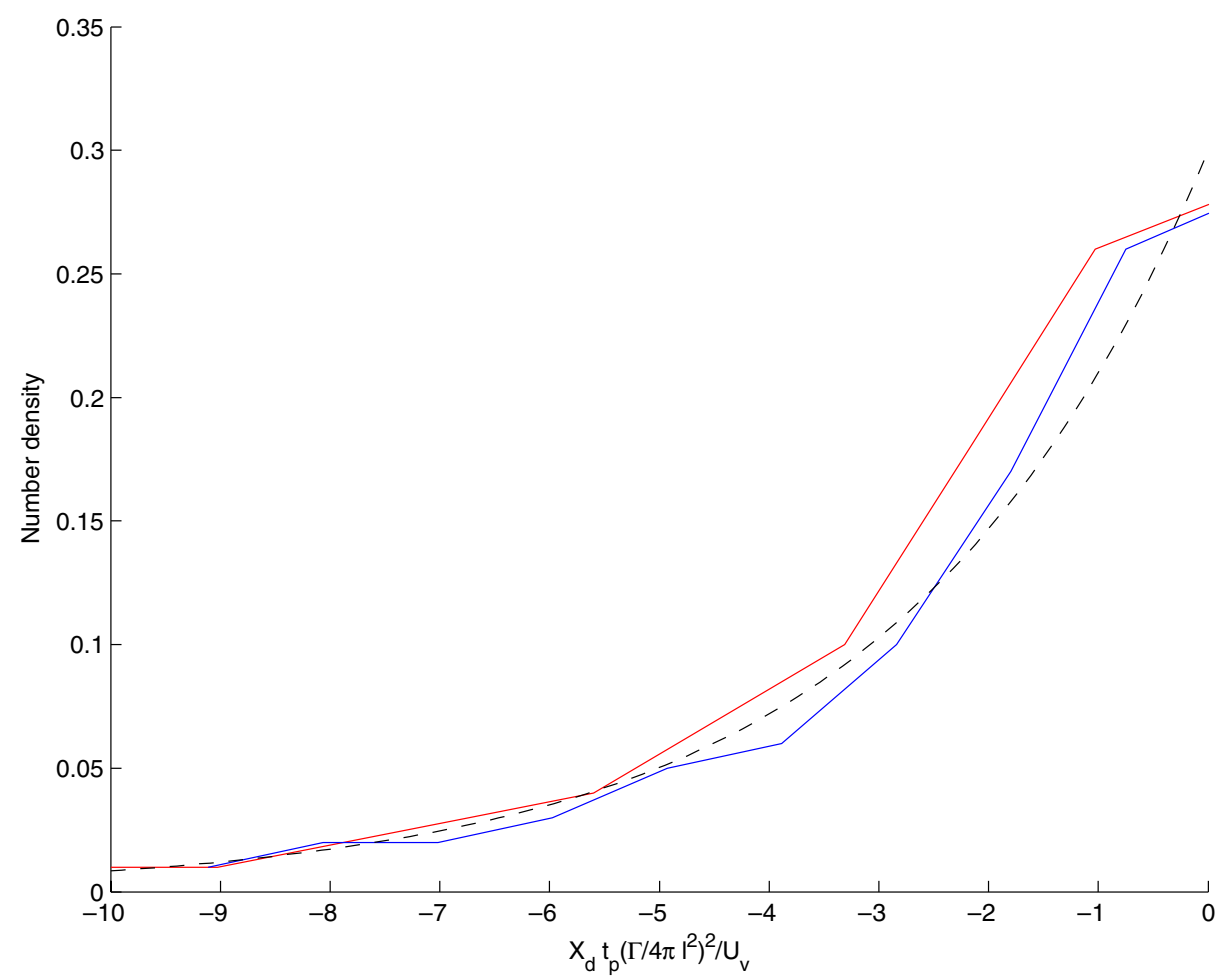

Fig. 7 Deposition profile of particles which initially lie within the shadow region of a Rankine vortex which is moving left to right. The number density corresponds to the fraction of the initial particles which deposited a distance $X_{d}$ from the initial starting point within the vortex. The particles are distributed uniformly along a line connecting the centre and the saddle point. The dashed curve corresponds to an exponential fit. The full curves correspond to numerical calculations for $v_{T} / U_{\omega}=$ 0.1 and 0.2 , where $l=1, \Gamma=1$ 

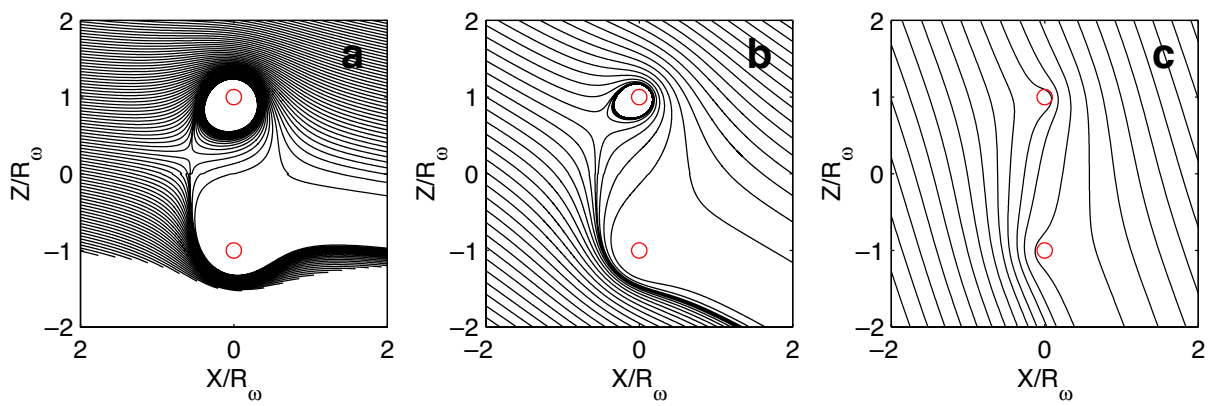

Fig. 8 Sedimentation of particles initially released above a propagating vortex $\operatorname{ring}$ for $l=0.1, \Gamma=1$, $R_{\omega}=1, \tau_{p}=0.01$ and $\mathbf{a} v_{T}=0.05$, b 0.2 , and $\mathbf{c} 0.9$. The particles are released in the plane $y=0$. Note how particles are excluded from the inner vortex and the significant shadow region around the structure

\subsubsection{Effect of vortex growing}

For laminar flow structures, the diffusion of vorticity causes the characteristic radius $l$ to grow as: $l^{2}=l_{0}^{2}+2 v t$. The vorticity in the core, which scales as $\Gamma / 4 \pi l^{2}$, therefore decreases, as does the maximum velocity which scales as $U_{m}=\Gamma / 2 \pi l$. The size of the region where particles are trapped scales as $R_{p s p} \sim \Gamma / 2 \pi v_{T}$ so after a time $\sim R_{p s p}^{2} / 2 v, l>R_{p s p}$ and the trapping region decreases in size. The time the particles remain trapped in the vortex scales as $4 \pi^{2} l_{0}^{4} / \Gamma^{2} t_{p}$. The particle residence time within the vortex is maximum when the time taken for particles to be expelled is comparable to the time the trapping region persists, ie $4 \pi^{2} l_{0}^{4} / \Gamma^{2} t_{p} \sim \Gamma^{2} / 8 \pi^{2} v_{T}^{2} v$, or

$$
\frac{\Gamma^{4}}{32 \pi^{4} g^{2} t_{p} v l_{0}^{4}} \sim 1,
$$

as confirmed by the numerical calculations of Fung [19].
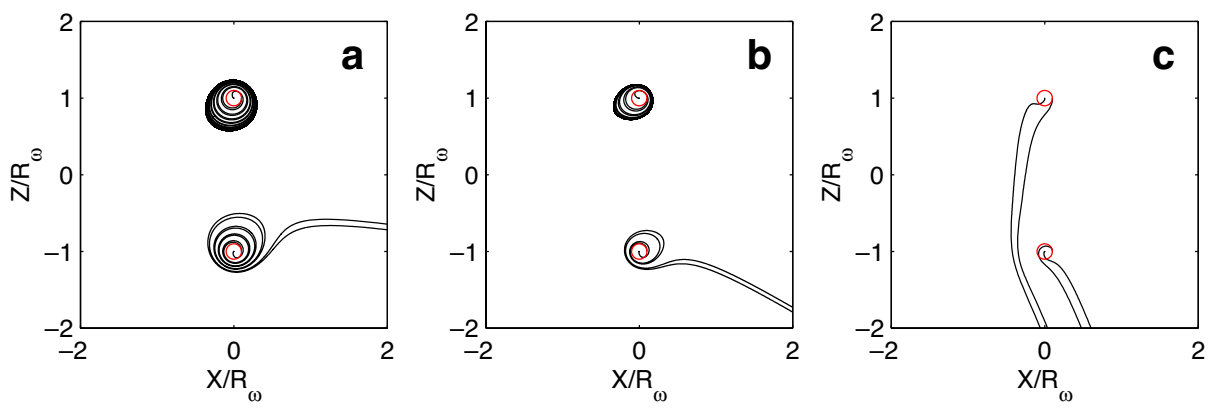

Fig. 9 Sedimentation of particles initially located in the core of a ring vortex, for $l=0.1, \Gamma=1$, $R_{\omega}=1, \tau_{p}=0.01$ and $\mathbf{a} v_{T}=0.05, \mathbf{b} 0.2$, and $\mathbf{c} 0.9$. The particle trajectories are plotted in the vortex frame of reference 


\subsection{Particle motions near steadily propagating vortex rings}

The calculations of Section 3.1 provide a leading order description of particle motion in and around the horizontal cores of a vortex ring. The flow generated by a vortex ring is described in the Appendix. From (35), the ejection time of particles from the vortex core scales as $4 \pi^{2} l^{4} /\left(\Gamma^{2} t_{p}\right)$ and is therefore very sensitive to the core radius, although the propagation speed $U_{\omega}$ is not.

Figure 8 shows the trajectories of particles starting above the vortex ring (where the particles are released above the vortex ring along $Y=0$ ). The trajectories are plotted in the frame moving with the vortex - in this frame, the increased inclination of the particle paths is due to their increasing fall velocity, from Fig. 8a to c. An excluded shadow region is created-the top portion of the vortex apparently shields the lower vortex from particles. Figure $8 \mathrm{~b}$ shows incident particles trapped in a path around the vortex ring, before they are ejected. For higher fall velocities, the PSP disappears. Figure 9 shows the paths of particles released within the vortex ring.

The horizontal distance $X_{d}$ travelled by dense particles released into the vortex core is plotted in Fig. 10 for varying $v_{T} / U_{\omega}$ and a fixed value of $S_{t}=U_{\omega} t_{p} / R_{\omega}$. The horizontal distance was defined as the horizontal distance travelled by the particles

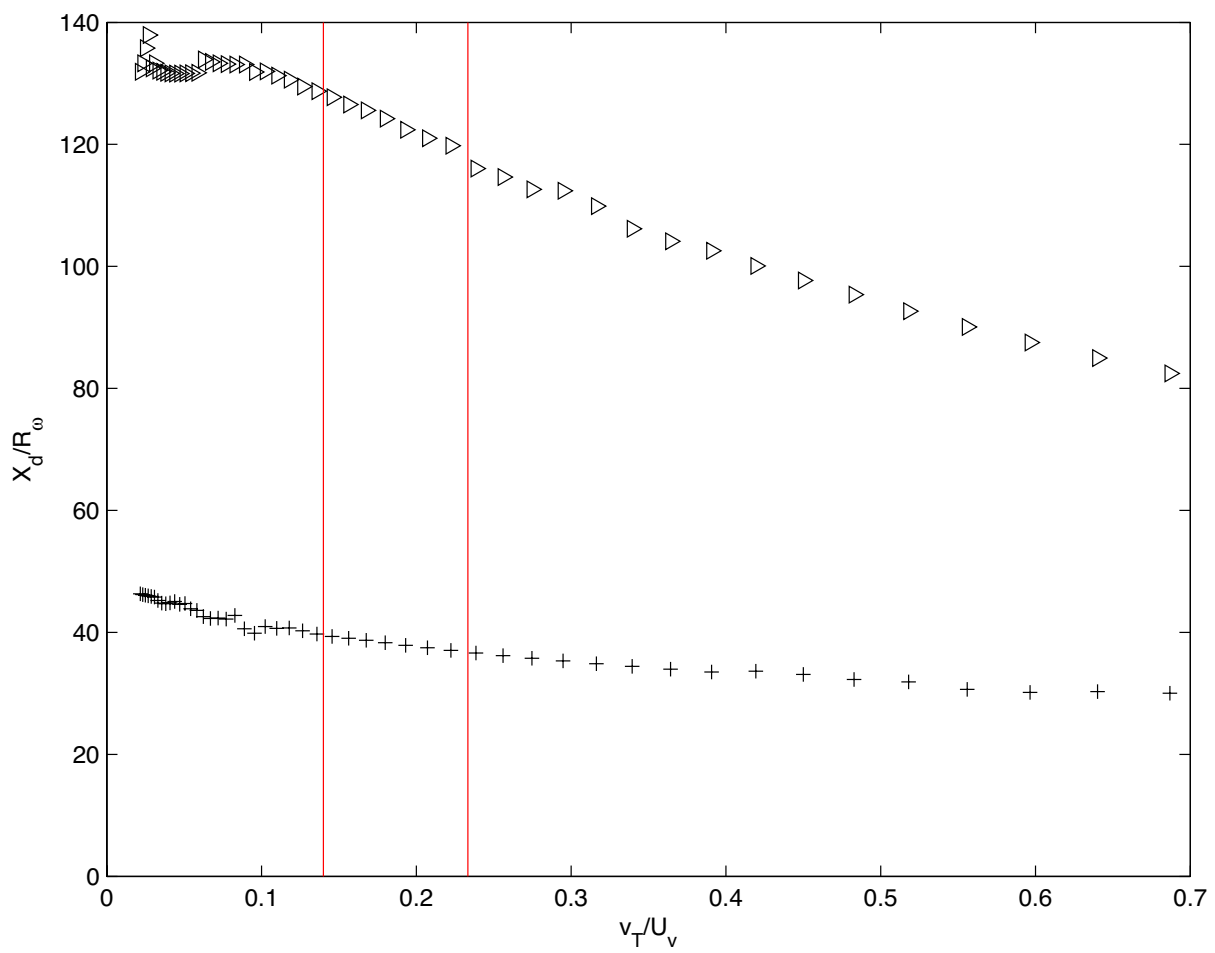

Fig. 10 Horizontal distances $X_{d}$ transported by dense particles before sedimenting to a surface $10 R_{\omega}$ below the vortex centre. The particles introduced at the core of the vortex ring (at $X=0, Z= \pm R_{\omega}$, $Y=0) . X_{d}$ plotted as a function of $v_{T} / U_{\omega} .+, \triangleright$ correspond to particles released in the lower and upper branch of the vortex. The particle Stokes number, $S_{t}=t_{p} U_{\omega} / R_{\omega}=120$, is comparable to the values in the experimental study described in Section 4. The vertical lines bound the range expected in the experimental study 
whilst falling through a vertical distance equal to $10 R_{\omega}$. The positions of particles introduced at the core of the vortex ring (at $X=0, Z= \pm R_{\omega}, Y=0$ ) are plotted as a function of $v_{T} / U_{\omega}$. The results are sensitive to the location of the rigid wall. The particle Stokes number in these calculations are chosen to be $S_{t}=t_{p} U_{\omega} / R_{\omega}=120$, which is comparable to those in the experimental study described in Section 4. The two vertical lines correspond to the range of $v_{T} / U_{\omega}$ in the experimental study.

\section{Laboratory Experiments}

A series of laboratory experiments were performed to compare with the theoretical models described in the previous sections. In order to provide results that are relevant to the medical application described in the introduction, the vortices were created by ejecting air through an orifice with the typical dimensions of the mouth $(3-8 \mathrm{~cm})$ with particles of similar density to water or blood.

\subsection{Experimental set-up}

\subsubsection{The vortex ring box}

A schematic of the experimental arrangements is shown in Fig. 11. The main component is a cylindrical hollow acrylic tube (internal diameter $31 \mathrm{~cm}$, length $45 \mathrm{~cm}$ ). An interchangeable rigid plate with various orifice shapes was fitted to the front of the cylinder. The back end of the cylinder was covered with an elastic sheet $2 \mathrm{~mm}$ thick. Deflecting the elastic sheet created a flow through the orifice, resulting in the generation of a vortex. The sheet was typically deflected by $1 \mathrm{~cm}$ over a duration of about $0.1 \mathrm{~s}$ when gently tapped, and $0.5 \mathrm{~s}$ when gently pushed. The amount of air expelled from the nozzle for a 1-cm deflection is approximately $250 \mathrm{~cm}^{3}$, typical of the tidal exchange volume of a childs' lung.

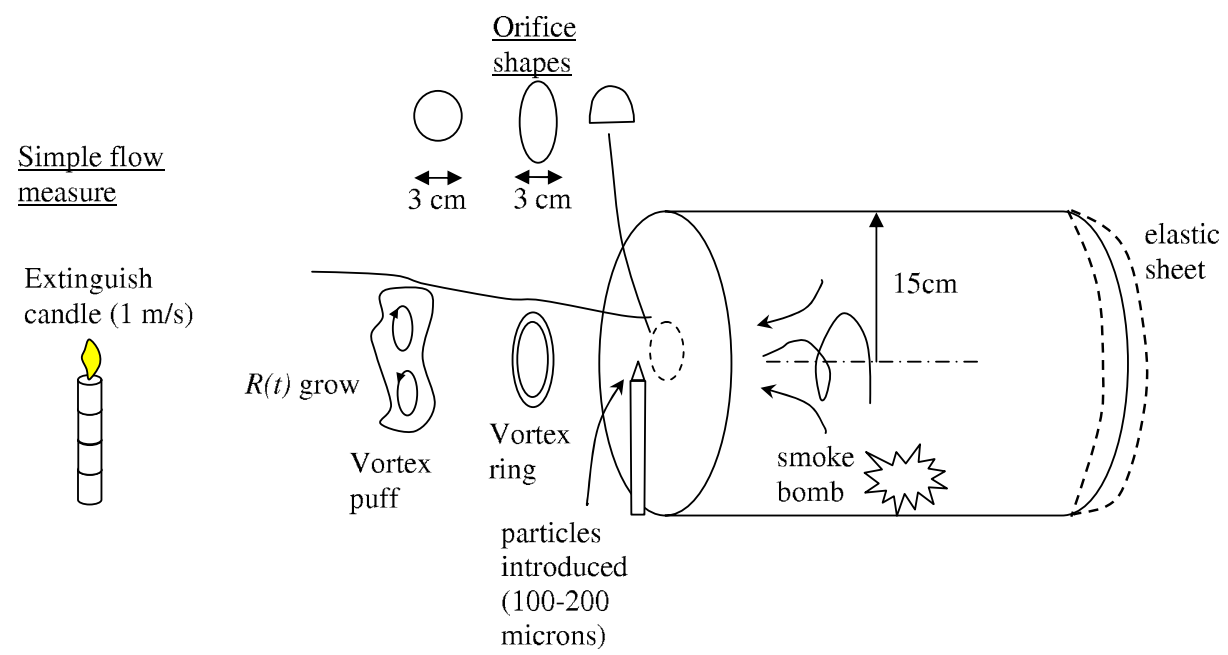

Fig. 11 Schematic of the experimental apparatus, illustrating the types of orifice shapes used. 
The vortex ring box is positioned so that the centre of the orifice was between $3-25 \mathrm{~cm}$ above a horizontal floor. The experiments were undertaken in a controlled environment with no significant background air motion; in one series of tests, a turbulent mean flow was introduced by placing a fan $1.5 \mathrm{~m}$ from the orifice blowing from the back side at an angle of about $30^{\circ}$ to the main axis.

\subsubsection{Smoke visualisation and particles}

To study the dispersion of small dense particles, we used dry milk powder (of density $\rho_{p}=1$ g.cm ${ }^{-3}$ ) whose size distribution, measured using a Malvern 2600D particle size analyser, is plotted in Fig. 12a. The mean particle diameter is $d_{p}=180 \mu \mathrm{m}$. The terminal fall speed was measured to be about $v_{T}=70-100 \mathrm{~cm} / \mathrm{s}$, consistent with the estimate based on Stokes settling velocity (which lies in the range $60-160 \mathrm{~cm} / \mathrm{s}$, for $\left.d_{p}=100-500 \mu \mathrm{m}\right)$. The particle response time is $t_{p}=v_{T} / g \approx 0.7-1 \mathrm{~s}$. The particle Stokes number, defined in relation to the vortex speed, is $S_{t}=U_{\omega} t_{p} / R_{\omega} \approx 100$ and $v_{T} / U_{\omega} \approx 0.15-0.3$.

The particles were placed in a small pile (4 $\mathrm{mm}$ high) on the top a thin vertical rod (of diameter $8 \mathrm{~mm}$ ) (see Fig. 12b). The top of the pile was located at various places in the orifice; mainly in the middle or near the edge. The deposition of the particles was visualised by placing black paper on the table. The vertical displacement of the particles was visualised by placing a black vertical plane parallel to the main axis of the cylinder, $40 \mathrm{~cm}$ to one side of it.

The vortex was visualised using 'smoke tablets' (Weco-Eitorf, Nr. 8105), which were ignited inside the vortex generator. To avoid the influence of buoyancy and residual motion in the plenum, the flow inside the apparatus was left to settle for about half a minute before performing the experiments. In some cases we produced intriguingly stable rings with visible net circulation around the ring axis (see Fig. 13), probably because some internal circulation in the vortex ring box remained when the experiment was started. The small size of the smoke particles (about $1 \mu \mathrm{m}$ ) means that over the course of the experiment, they will not typically be ejected from the
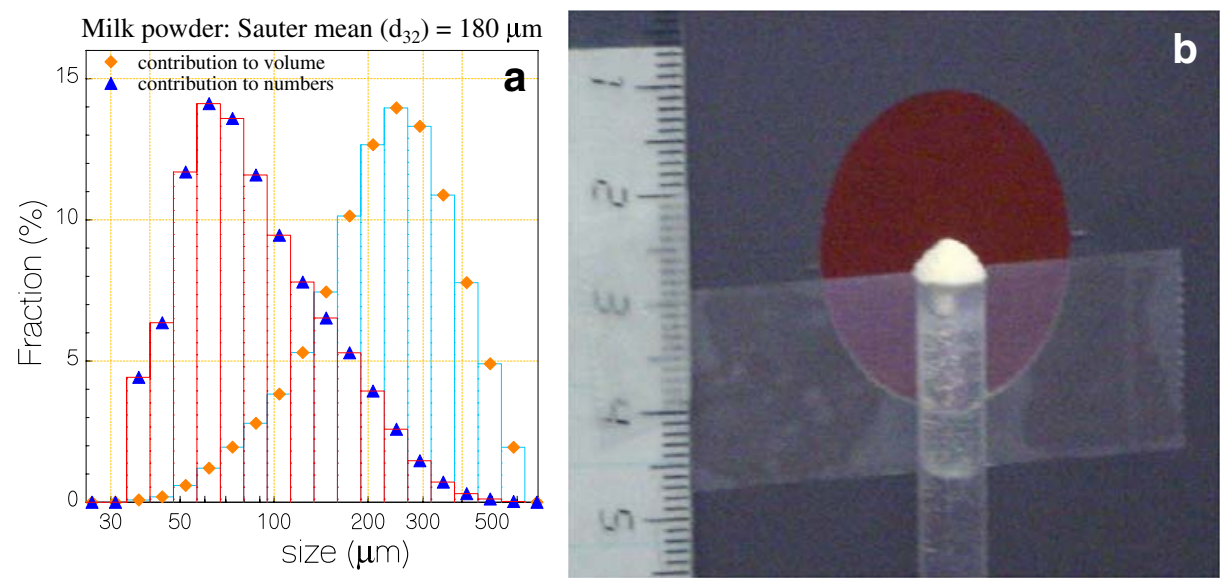

Fig. 12 The size distribution of milk powder is shown in a. A photograph detailing the particle pile placed in front of a semi-circular orifice is shown in $\mathbf{b}$ 
Fig. 13 Elevated view of a vortex ring, with net swirl, emitted from the vortex generator and visualised with smoke. The background image shows the vortex generator

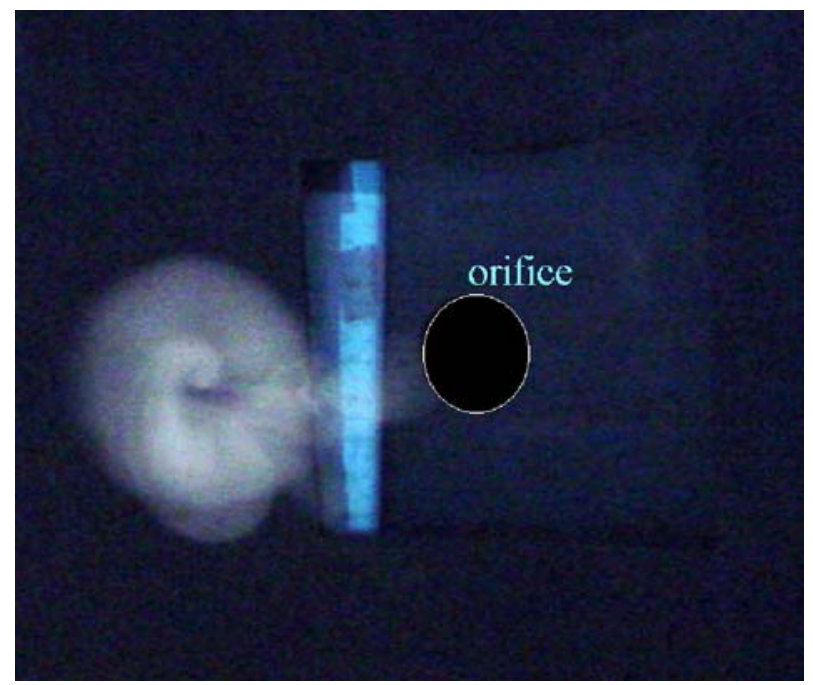

vortex core. The smoke pattern and particle deposition were recorded using a colour digital camera (Sony DSC-P5) fixed on a tripod, giving a resolution of $2048 \times 1360$ pixels for photographs and a lower resolution of $320 \times 240$ pixels, at $25 \mathrm{fps}$.

\subsection{Experimental results}

\subsubsection{Vortex speed}

Vortices generated from a circular orifice produced thick circular vortex 'rings' or 'vortex puffs'; a typical front and side view is shown in Fig. 13. The vortex speed was estimated from a frame-by-frame analysis of the video images and was typically $U_{\omega}=300-500 \mathrm{~cm} / \mathrm{s}$.

A volume of air $V_{a}=250 \mathrm{~cm}^{3}$ was ejected over a period of $\Delta t=0.2 \mathrm{~s}$ through a hole of radius $R_{h}=1.5 \mathrm{~cm}$. The characteristics of the vortex can be estimated using the concepts described in Section 2, where the circulation, impulse and $K E$ are conserved during the adjustment phase (see [45]). To understand this, we approximate the initial state of the flow (generated by the exit from the hole) as a cylindrical vortex sheet of radius $R_{h}$ and circulation $\Gamma=U_{a} L_{a}$, where $L_{a}=V_{a} / \pi R_{h}^{2}$ is the length of the slug and $U_{a}=L_{a} / \Delta t$ is the flow through the orifice. The flow generated by the forcing generates an impulse $I_{M}=\rho U_{a} V_{a}$, kinetic energy of the flow is $K E=\frac{1}{2} \rho U_{a}^{2} L_{a} \pi R_{h}^{2}$, and the total circulation (assuming that a cylindrical vortex sheet is created) is $\Gamma=\left(V_{a} / \pi R_{h}^{2}\right)^{2} / \Delta t$. From the conservation of impulse, the final state of the inviscid vortex should be a ring vortex whose curvature is $R_{\omega}=R_{h}$. From the conservation of energy, the speed of the vortex is $U_{\omega}=\left(V_{a} / \pi R_{h}^{2}\right)^{2} /\left(4 \pi R_{a} \Delta t\right)\left(\pi^{2} R_{h}^{2} / V_{a}+\frac{3}{2}\right) \approx$ $300-500 \mathrm{~cm} / \mathrm{s}$ (for $\Delta t=0.1-0.2 \mathrm{~s}$ ). The vortex core radius is estimated to be approximately $l / R_{\omega}=8 \exp \left(-\pi^{2} R_{h}^{3} / V_{a}\right) \approx 1.1$, ie comparable with the radius of curvature. The conservation of energy, impulse and circulation provide insight into the adjustment process of the vortex. Since the slug of air $V_{a}$ ejected from the cylinder is much larger than the enclosed volume of the vortex $V_{c}$ (typically $V_{a} / V_{c} \geq 50$ ), a large volume of smokey air will be detrained from the vortex during the adjustment 

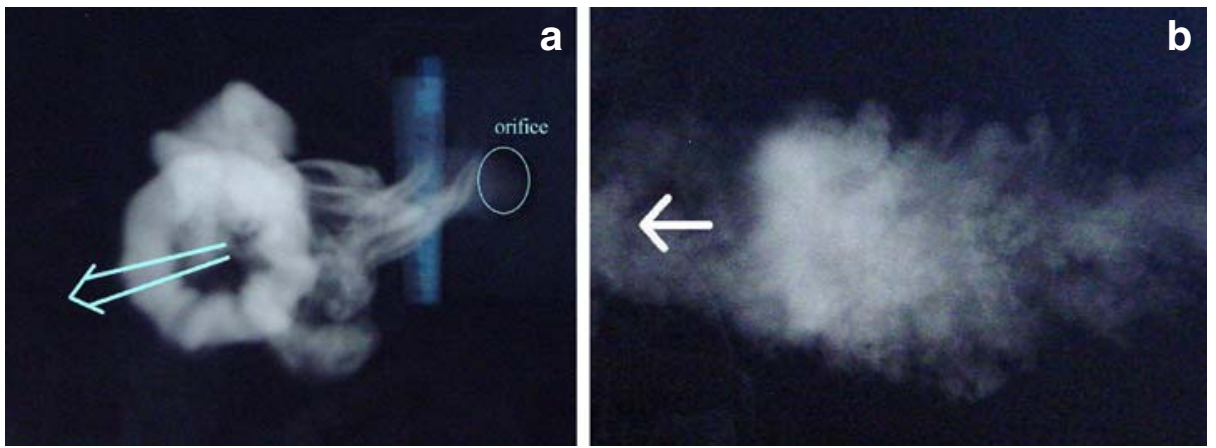

Fig. 14 a Vortex ring with smoke, front view. b Vortex puff with smoke, side view

process. The vorticity associated with the detrained air is weak compared with the vorticity inside the vortex.

The vortex grew from 4 to $8 \mathrm{~cm}$ in diameter as it moved from the orifice to a distance $100 \mathrm{~cm}$ away. This is consistent with the estimates of the adjustment process, since although the core will be comparable with the hole diameter, the vortex has a final diameter of $2\left(R_{\omega}+l\right) \approx 5.5 \mathrm{~cm}$, even before entrainment is taken into account. This physical interpretation is further supported by the fact that the vortex speed hardly changes during this initial phase-if entrainment were responsible for the increase in diameter (by a factor of 2), there would have been a corresponding decrease (by a factor of 10) in the speed of translation.

Since the vortex Reynolds number is $\operatorname{Re}_{v}=2 U_{\omega} R_{\omega} / v \sim 8000$, the core is turbulent. The internal turbulent motion disperses smoke throughout the internal core. This is a more vigorous kind of motion than the delicate, slow moving 'smoke rings' produced by smokers, in which there are no small-scale eddies to disperse the smoke. The side view (Fig. 14a,b) indicates that the ring loses smoke by turbulent transport over its boundaries.

\subsubsection{Effect of orifice shape}

The shape of the orifice had a significant effect on the vortex. In the first case, the circular orifice was replaced by an elliptical orifice of aspect ratio 1:1.85; the large and small diameters being 11.5 and $6.2 \mathrm{~cm}$, respectively. The elliptical ring oscillated as it moved forward and the internal motion became significantly more turbulent than the circular vortex ring. This is well-known and is caused by the varying curvature of the vortex loop in the vortex ring [35], see also Section 2.2.

In the second case, the orifice was semi-circular. The vortex became significantly distorted as well as oscillating and becoming more turbulent. Some elements of the vortex loop, especially near the sides moved upwards (carrying smoke with them) while the central lower part of the vortex descends. These effects are caused by the strong variation of the curvature of the loop varying from a tight curve in the corners to being only slightly curved elsewhere. The difference with the previous case of the elliptical vortex is that here the curvature is different on the top and bottom halves of the vortex, and the curvature at the corners is not symmetric with respect to the horizontal axis. 


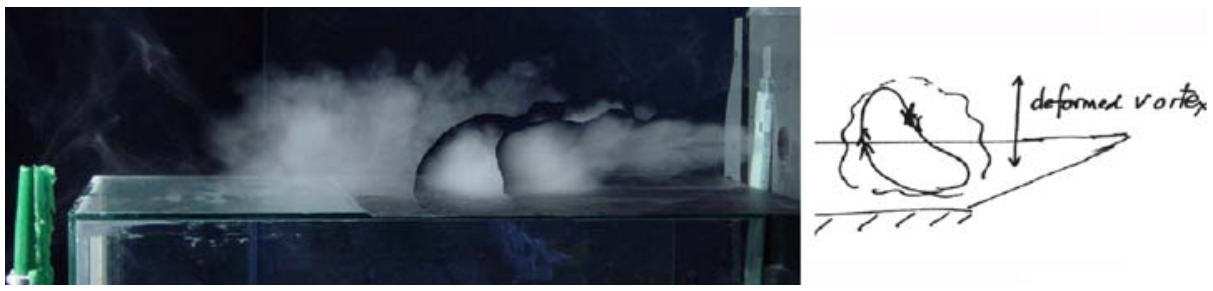

Fig. 15 Photograph showing three superimposed images of a smoke vortex ring colliding with a rigid plane surface. They show the smoke ring bending down towards a rigid floor, and lifting up again as a puff of smoke. A schematic of the vortex deformation as it interacts with the floor is shown on the right

\subsubsection{Effect of a wall}

In another modification, a wall was placed just below the orifice, parallel to the axis of the cylinder. This situation is similar but not identical to two parallel vortex rings in open space. As known from literature [34], at higher Reynolds numbers, the friction with the wall is less important than the interaction with the 'image' vortex, which in this case slows down the part of the ring nearest the wall. As a result, the trajectory of the ring bends towards the wall. Whereas with two parallel vortex rings the touching parts can merge ('reconnection'), leading to a single, larger vortex ring. However, with a solid wall, the vortex further tilts towards the wall, and after much internal motion bounces off again, as shown in Fig. 15.

\subsubsection{Effect of external turbulence}

Next the effects of external turbulent air stream were studied. The strength and nature of this stream was assessed qualitatively by placing a candle on the axis. The flame was fluctuating and was close to extinction, indicating that the velocity of the imposed air stream was about $1 \mathrm{~m} / \mathrm{s}$ or larger. When a vortex was propelled into this disturbed air flow it was quite noticeable from the smoke flow visualization that the vortex ring/puff was more turbulent and disorganized, i.e. the eddying motions in the puff were stronger and larger scale than without the external airstream. The diameter of the ring/puff increased to about $20 \mathrm{~cm}$ at a distance $50 \mathrm{~cm}$ from the orifice.

Finally, disturbed flows were also considered when the vortices and jet impacted onto a vertical plate placed perpendicular to the path of the vortex. The dimensions of the plate were $75 \mathrm{~cm}$ high (above the table) and $35 \mathrm{~cm}$ wide. The strength of the vortex reaching the plate was gauged by the fact that it extinguished a candle placed about $5 \mathrm{~cm}$ in front of the plate. This means that the velocity of the air flow was about 1-2 $\mathrm{m} / \mathrm{s}$. The vortex motion was visualized with smoke. This showed that diameter of the vortex ring increased to about $12-15 \mathrm{~cm}$ as it impacted onto the plate. In the absence of the plate the diameter was about $8 \mathrm{~cm}$.

\subsubsection{Particle dispersion and deposition}

The particles were observed to be resuspended only when the vortex core passed over them. The white particles were deposited onto black paper placed on the workbench. Few particles were found between the orifice and about $0.4 \mathrm{~m}$ along the 
axis, most lay between 0.4 and $0.6 \mathrm{~m}$, but a significant number were carried beyond $0.8 \mathrm{~m}$. A striking feature of the distribution was that the width of the strip of particles on the black paper grew quite markedly from about $0.1 \mathrm{~m}$ to about $0.2 \mathrm{~m}$ at the critical distance of $0.4-0.5 \mathrm{~m}$ downwind. This is consistent with the numerical results in Fig. 10, where the particles are entrained by the lower portion of the vortex ring (the vertical lines show the range of particle fall velocity). It appears that the eddying in the vortex throws the particles outwards (as in the experiments of Perkins et al. [39]). Observation of the particle movement against the background of a vertical black sheet showed that a significant number of particles travelled with the vortex over the distance $0.6-0.8 \mathrm{~m}$ and are thrown upwards, sideways and downwards. The fact that the fall speed (about $0.7-1.0 \mathrm{~m} / \mathrm{s}$ ) is less than the vortex speed shows that the distribution of particles on the ground is mainly determined by the eddying of the vortex.

When the orifice diameter is changed no great change occurs in the particle distribution. When the shape is changed from circular to elliptical the particle deposition increases. However when the shape is changed to semicircular the particles are deposited on the ground from below the orifice to about $0.5 \mathrm{~m}$ down wind, with a few particles travelling to $0.7 \mathrm{~m}$ (there is no maximum concentration at $0.5 \mathrm{~m}$ as with the circular orifice). Particles on the centre line of the vortex were carried upwards by the upward moving elements of the vortex that occur with such a distorted vortex. In this case the width of the strip of deposited particles does not suddenly increase, as with the circular jet. It remains at about $10-15 \mathrm{~cm}$ along the axis.

The presence of weak ambient winds (of about $1 \mathrm{~m} / \mathrm{s}$ ) affects the particle dispersion. The width of the strip is not only greater (at about $20 \mathrm{~cm}$ ), but it steadily increases with downwind distance. This is associated with the vortex ring/puff being a wholly turbulent puff with the vortex ring element of the structure having been destroyed. Nevertheless particles are transported by this turbulent flow as the deposition data demonstrates.

The presence of a plate placed across the path of the vortex (at $75 \mathrm{~cm}$ from the orifice) also affects the dispersion of particles. The impact of particles was measured by spraying the surface with a light lubricating oil. It was found that with a strong circular vortex (i.e. one that extinguished a candle) the particles impacted onto the plate over a diameter of $15 \mathrm{~cm}$. The height of this impact circle was about $15 \mathrm{~cm}$. In other words its average height was only about $5 \mathrm{~cm}$ lower than the orifice. With a weaker vortex the particles tended to settle out more strongly; most particles were spread over a strip of about $15 \mathrm{~cm}$ wide. The strip extended vertically from the level of the table up to about $10 \mathrm{~cm}$ above it. When a semicircular orifice was used this caused some of the particles to rise higher and also impact onto the plate at a higher level.

\section{Conclusion}

The analysis in this paper of isolated vortical structures shows that their main flow features can be described in terms of general dynamical and, in some cases, topological concepts about the local and overall properties of flows, such as the strain/vorticity ratio, the helicity, the genus of vortical surfaces, and an important new parameter-the ratio of the kinetic energy of the variance to that of the bulk flow that tends to increase during their life time. The latter property shows how the 
detailed profiles of vortical structures tend to disappear over their lifetime. It has been shown here that these concepts are applicable to non-ideal structures, including the effects of rigid boundaries and other disturbances, as our laboratory experiments on simple and complex vortices have largely confirmed.

Further research with more detailed numerical simulations are needed to test the concepts proposed here and their robustness in more complex flows, and with various initial conditions. Theoretical studies might be able to extend our understanding of which kinds of structure are steady and stable (see the reviews in Hunt and Vassilicos [25]). Many of these questions are of great practical importance, for instance, mixing in engines [36].

Increasingly it is realised that the properties of the large scale eddies of homogenous turbulent flows may depend as much on the dynamics and integral properties of individual vortical structures and on the local and global interactions between them (cf $[12,38])$ rather than on the tendency towards statistical equilibrium that might be insensitive to such dynamics [33]. If the former mechanism is significant, research is clearly needed to necessary to calculate the aggregate properties of all the structures in order to compare with statistical measurements and theory.

The results presented here on inertial particles in vortical structures have been referred to previously in the literature. But the general analysis developed here based on considering the combined effects of vorticity and strain provides a physical concept for understanding inertial particle motions in quite complex flows, such as strained vortices in turbulence. The next phase of this research involves estimating how, as the particles become concentrated, their motions through the fluid can significantly affect the distribution of mean vorticity in the structures [31].

Acknowledgements We are grateful for the inspired leadership of Frans Nieuwstadt at Technological University of Delft who particularly encouraged research on dynamics and flow processes in vortical structures in every kind of turbulent and non-turbulent flow. JCRH is also grateful for support from Cornell University where he was a Mary Upson visiting Professor between 2003 and 2006, and many fruitful conversations about vortex dynamics with Professor Sid Leibovich. IE gratefully acknowledges support from the Engineering and Physical Sciences Research Council (EP/E029302/1) and a Phillip Leverhulme Prize (2005). IE is also grateful to Professor George Bergeles for hosting him at National Technical University of Athens.

\section{Appendix}

Here we consider the dynamics of particles released near to a ring vortex of curvature $R_{\omega}$ with a core radius $l$ and circulation $\Gamma$. The Stokes streamfunction, $\Psi_{f}$, describing the flow in the frame moving with the vortex is

$$
\Psi_{f}=\frac{\Gamma\left(y R_{\omega}\right)^{\frac{1}{2}}}{2 \pi}\left(\left(\frac{2}{k}-k\right) K(k)-\frac{2}{k} E(k)\right), \quad k^{2}=\frac{4 y R_{\omega}}{x^{2}+\left(y+R_{\omega}\right)^{2}}
$$

where $K$ and $E$ are elliptic functions of the first and second kind. The self-induced velocity of the vortex is $U_{\omega}=\Gamma / 4 \pi R_{\omega}\left(\log \left(8 R_{\omega} / l\right)-1 / 2\right)$. The flow induced is $u_{x}=-1 / y \partial \psi / \partial y, u_{y}=1 / y \partial \psi / \partial x$. 


\section{References}

1. Alcan: Liquid metal launder. US Patent 4958809 (1989)

2. Bajer, K., Moffatt, H.K.: On a class of steady confined Stokes flows with chaotic streamlines. J. Fluid Mech. 212, 337-363 (1990)

3. Bagnold, R.A.: Physics of Blown Sand and Desert Dunes. Marrow, Lodz (1941)

4. Batchelor, G.K.: An Introduction to Fluid Mechanics. Cambridge University Press, Cambridge (1967)

5. Batchelor, G.K., Nitsche, J.M.: Expulsion of particles from a buoyant blob in a fluidized bed. J. Fluid Mech. 278, 63-81 (1994)

6. BBC: Case turned on 158 spots of blood. http://news.bbc.co.uk/2/hi/uk_news/england/southern_ counties/4661302.stm (2006)

7. Berger, M.: Lecture to Lighthill Institute of Mathematical Sciences. London (2006)

8. Chen, L., Goto, S., Vassilicos, J.C.: Turbulent clustering of stagnation points and inertial particles. J. Fluid Mech. 553, 143-154 (2006)

9. Chung, J.N., Trout, T.R.: Simulation of particle dispersion in an axisymmetric jet. J. Fluid Mech. 186, 199-222 (1988)

10. Dabiri, J.O., Colin, S.P., Costello, J.H., Gharib, M.: Flow patterns generated by oblate medusan jellyfish: field measurements and laboratory analyses. J. Exp. Biol. 208, 1257-1265 (2005)

11. Davidson, P.A.: Turbulence: An Introduction for Scientists and Engineers. Oxford University Press, p. 680. (2006)

12. Davidson, P.A.: On the large-scale structure of homogeneous, two-dimensional turbulence. J. Fluid Mech. 580, 431-450 (2007)

13. Davila, J., Hunt, J.C.R.: Settling of small particles near vortices and in turbulence. J. Fluid Mech. 440, 117-145 (2001)

14. Eames, I., Dalziel, S.B.: Dust resuspension by the flow around an impacting sphere. J. Fluid Mech. 403, 305-328 (2000)

15. Eames, I., Flor, J-B.: Fluid transport by dipolar vortices. Dyn. Atmos. Ocean. 28, 93-105 (1998)

16. Eames, I., Gilbertson, M.A.: The settling and dispersion of small dense particles by spherical vortices. J. Fluid Mech. 498, 183-203 (2004)

17. Eames, I., Hunt, J.C.R., Belcher, S.E.: General theory for inviscid flow through clouds of bodies. J. Fluid Mech. 515, 371-389 (2004)

18. Flegg, G.H.: From Geometry to Topology. Grane, Russak \& Company, New York (1974)

19. Fung, J.C.H.: Residence time of inertial particles in a vortex. J. Geophys. Res. 105, 14261-14272 (2000)

20. Fung, J.C.H., Hunt, J.C.R, Perkins, R.J., Wray, A.A., Stretch, D.: Defining the zonal structure of turbulence using the pressure and invariants of the deformation tensor. In: Johansson, A.V., Alfredsson, P.H. (eds.) Advances in Turbulence, vol. 3, pp. 395-404. Springer, Berlin Heidelberg New York (1991)

21. Gilbertson, M.A., Yates, J.G.: The motion of particles near a bubble in a gas-fluidized bed. J. Fluid Mech. 323, 377-385 (1996)

22. Hawthorne, W.R.: Wright Memorial Lecture. A.I.A.A. (1975)

23. Hunt, J.C.R.: Vorticity and vortex dynamics in complex turbulent flows. Trans. Can. Soc. Mech. Eng. 11, 21-35 (1987)

24. Hunt, J.C.R., Eames, I.: The disappearance of laminar and turbulent wakes in complex flows. J. Fluid Mech. 457, 111-132 (2002)

25. Hunt, J.C.R., Vassilicos, J.C. (eds.): Turbulence Structure and Vortex Dynamics. Cambridge University Press, Cambridge (2001)

26. Hunt, J.C.R., Abell, C.J., Peterka, J.A., Woo, H.: Kinematical studies of the flows around free or surface-mounted obstacles; applying topology to flow visualization. J. Fluid Mech. 86, 179-200 (1978)

27. Hunt, J.C.R., Perkins, R.J., Fung, J.C.H.: Review of the problems of modelling disperse twophase flows. In: Hewitt, G.F., Kim, J.H., Lahey, R.T., Delhaye, J.M., Zuber, N. (eds.) Multiphase Science and Technology. Two-phase Flow Fundamentals, vol. 8, pp. 595-643. Begell House, New York (1995)

28. Hunt, J.C.R., Eames, I., Westerweel, J.: Mechanics of inhomogeneous turbulence and interfacial layers. J. Fluid Mech. 554, 499-523 (2006)

29. Husain, H.S., Hussain, F.: The elliptic whistler jet. J. Fluid Mech. 397, $23-41$ (1999) 
30. Joia, I.A., Ory, E., Perkins, R.J.: A discrete vortex model of particle laden jets. Paper presented at the 3rd International Conference on Multiphase Flows, Lyon (1998)

31. Kiger, K.T., Lasheras, J.C.: Dissipation due to particle/turbulence interaction in a two-phase, turbulent shear layer. Phys. Fluids. 9, 3005-3023 (1997)

32. Lamb, H.: Hydrodynamics. Dover, New York (1932)

33. Landau, L.D., Lifshitz, E.M.: Fluid Mechanics. Pergamon, New York (1959)

34. Lim, T.T., Nickels, T.B., Chong, M.S.: A note on the cause of rebound in the head-on collision of a vortex with a wall. Exp. Fluids 12, 41-28 (1991)

35. Lugt, H.J.: Vortex Flow in Nature and Technology. Wiley-Interscience, New York (1983)

36. Lumley, J.L.: Early work on fluid mechanics in the IC engine. Ann. Rev. Fluid Mech. 33, 319-338 (2001)

37. Marcu, B., Meiburg, E., Newton, P.K.: Dynamics of heavy particles in a Burgers vortex. Phys. Fluids. 7, 400-410 (1995)

38. Moffatt, H.K., Kida, S., Ohkitani, K.: Stretched vortices-the sinews of turbulence; largeReynolds-number asymptotics. J. Fluid Mech. 259, 241-264 (1994)

39. Perkins, R.J., Ghosh, S., Phillips, J.C.: Interaction of particles and coherent structures in a plane turbulent air jet. In: Johansson, A.V., Alfredsson, P.H. (eds.) Advances in Turbulence, vol. 3, pp. 93-100. Springer, Berlin Heidelberg New York (1991)

40. Reynolds, W.C., Parekh, D.E, Juvet, P.J.D., Lee, M.J.D.: Bifurcating and blooming jets. Ann. Rev. Fluid Mech. 35, 295-315 (2003)

41. Saffman, P.G.: Vortex Dynamics. Cambridge University Press, Cambridge (1992)

42. Shariff, K., Leonard, A.: Vortex rings. Ann. Rev. Fluid Mech. 24, 235-279 (1992)

43. Stamhuis, E.J., Nauwelaerts, S.: Propulsive force calcuations in swimming frogs II. Application of a vortex ring model to DPIV data. J. Exp. Biol. 208, 1445-1451 (2005)

44. Tang, J., Li, Y., Eames, I., Chang, C., Ridgway, G.: Factors involved in the aerosol transmission of infection and control of ventilation in healthcare premises. J. Hosp. Infect. 64, 100-114 (2006)

45. Taylor, G.I.: Formation of a vortex ring by giving impulse to a circular disk and then dissolving it away. J. Appl. Phys. 24, 104-105 (1953)

46. Townsend, A.A.: The Structure of Turbulent Shear Flows, 2nd edn. Cambridge University Press, Cambridge (1976)

47. Turner, J.: Buoyancy Effects in Fluids. Cambridge University Press, Cambridge (1973)

48. Vincent, A., Meneguzzi, M.: The spatial structure and statistical properties of homogeneous turbulence. J. Fluid Mech. 225, 1-20 (1991)

49. Voropayev, S.: Private Communication (2007)

50. Weigand, A., Gharib, M.: On the decay of a turbulent vortex ring. Phys. Fluids 6, 3806-3808 (1994)

51. Westerweel, J., Pedersen, J.M., Fukushima, C., Hunt, J.C.R.: Mechanics of the turbulent/ non-turbulent interface of a jet. Phys. Rev. Lett. 95, 174501 (2005) 